University of Nebraska - Lincoln

DigitalCommons@University of Nebraska - Lincoln

$12-2009$

\title{
Perturbation theory analysis of attosecond photoionization
}

Anthony F. Starace

University of Nebraska-Lincoln, astarace1@unl.edu

Evgeny A. Pronin

University of Nebraska - Lincoln, epronin2@unl.edu

M. V. Frolov

Voronezh State University, Russia, frolov@phys.vsu.ru

N. L. Manakov

Voronezh State University, Universitetskaya pl. 1, Voronezh, 394006 Russia, manakov@phys.vsu.ru

Follow this and additional works at: https://digitalcommons.unl.edu/physicsstarace

Part of the Physics Commons

Starace, Anthony F.; Pronin, Evgeny A.; Frolov, M. V.; and Manakov, N. L., "Perturbation theory analysis of attosecond photoionization" (2009). Anthony F. Starace Publications. 170.

https://digitalcommons.unl.edu/physicsstarace/170

This Article is brought to you for free and open access by the Research Papers in Physics and Astronomy at DigitalCommons@University of Nebraska - Lincoln. It has been accepted for inclusion in Anthony F. Starace Publications by an authorized administrator of DigitalCommons@University of Nebraska - Lincoln. 


\title{
Perturbation theory analysis of attosecond photoionization
}

\author{
E. A. Pronin and Anthony F. Starace \\ Department of Physics and Astronomy, The University of Nebraska, Lincoln, Nebraska 68588-0111, USA
}

\author{
M. V. Frolov and N. L. Manakov \\ Department of Physics, Voronezh State University, Voronezh 394006, Russia
}

(Received 30 September 2009; published 1 December 2009)

\begin{abstract}
Ionization of an atom by a few-cycle attosecond xuv pulse is analyzed using perturbation theory (PT), keeping terms in the transition amplitude up to second order in the pulse electric field. Within the PT approach, we present an $a b$ initio parametrization of the ionized electron angular distribution (AD) using rotational invariance and symmetry arguments. This parametrization gives analytically the dependence of the AD on the carrier envelope phase (CEP), the polarization of the pulse, and on the ionized electron momentum direction, $\hat{\mathbf{p}}$. For the general case of an elliptically polarized pulse, we show that interference of the first- and secondorder transition amplitudes causes a CEP-dependent asymmetry (with respect to $\hat{\mathbf{p}} \rightarrow-\hat{\mathbf{p}}$ ) and both elliptic and circular dichroism effects. All of these effects are maximal in the polarization plane and depend not only on the CEP but also on the phase of dynamical atomic parameters that enter our parametrization of the AD. Within the single active electron model of an atom, for an initial $s$ or $p$ state we define all dynamical parameters in terms of radial matrix elements (analytic expressions for which are given for the Coulomb and zero-range potentials). For ionization of the $\mathrm{H}$ atom by linearly polarized pulses, our PT results are in excellent agreement with results of numerical solutions of the time-dependent Schrödinger equation of Peng et al. [New J. Phys. 10, 025030 (2008)]. Also, our numerical results show that the asymmetries and dichroism effects at low electron energies have a different physical origin from those at high electron energies. Moreover, our results for Gaussian and cosine-squared pulse shapes are in good qualitative agreement. Finally, we show that our analytic formulas may prove useful for determining few-cycle extreme ultraviolet (xuv) pulse characteristics, such as the CEP and the polarization.
\end{abstract}

DOI: $10.1103 /$ PhysRevA.80.063403

PACS number(s): 32.80.Fb, 32.80.Rm, 32.80.Qk

\section{INTRODUCTION}

Rapid progress in the generation of ultrashort laser pulses over the past decade has enabled experimentalists to produce few-cycle laser pulses in both the femtosecond ir [1] and the attosecond extreme ultraviolet (xuv) [2-4] temporal and frequency domains with stable (and even tunable) carrier envelope phases (CEPs). The ultrashort duration of even manycycle xuv attosecond pulses makes them an ideal tool for probing electron motion in atoms and molecules [4-8], for imaging molecular orbitals [9], and for measuring the instantaneous electric field of a few-cycle ir pulse $[10,11]$. The successful use of an attosecond xuv pulse in such pumpprobe applications requires knowledge of the dependence of its interaction with matter on the pulse parameters. For nearly all experimental applications up to now, the only important parameters for a typically weak xuv attosecond pulse have been the carrier frequency and the pulse's duration and polarization. The experimental achievement of few-cycle attosecond pulses having ultra short duration $(\sim 100$ as $)$ and large bandwidth $(\sim 10 \mathrm{eV})$ requires an understanding of the effects of additional pulse characteristics, such as the pulse shape and the CEP, i.e., the phase between the maxima of the pulse envelope and the monochromatic carrier wave. These characteristics will become of crucial importance when the intensity of attosecond xuv pulses increases by about one order of magnitude beyond current experimental capabilities (i.e., to intensities of order $\sim 10^{15} \mathrm{~W} / \mathrm{cm}^{2}$ ) so that nonlinear effects become significant [12]. Even for intensities up to $\sim 10^{16} \mathrm{~W} / \mathrm{cm}^{2}$, however, the interaction of xuv pulses with atoms and molecules can be treated with high accuracy using perturbation theory (PT) [13-15]. Note that the importance of CEP effects for few-cycle ir pulses has been demonstrated experimentally [16-18] and has been the subject of theoretical works focused on above-threshold ionization (ATI) $[19,20]$, high-order harmonic generation (HHG) [21], and multiphoton excitation and ionization [22,23]. In particular, Cormier and Lambropoulos [19] have analyzed numerically the dependence of the asymmetry of the ionized electron angular distribution on the CEP at electron energies between neighboring ATI peaks and explained these asymmetries in terms of the interference between the transition amplitudes for successive ATI peaks. Moreover, a perturbative analysis in Ref. [19] resulted in an analytic expression for the CEP dependence. Retrieval of the CEP of a few-cycle ir laser pulse from asymmetries in the ionized electron angular distributions has also been the subject of recent experimental investigations $[17,18]$. A general formulation of CEP effects (for the case of linearly polarized pulses) by Roudnev and Esry [24] has shown that these effects originate from the interference between different photon amplitudes. In addition, it has been shown in Ref. [12] by direct numerical solution of the time-dependent Schrödinger equation (TDSE) that the asymmetry in the angular distribution of electrons ionized by an attosecond xuv pulse depends on the pulse intensity as $I^{3 / 2}$. This fact supports the idea that such asymmetries originate from the interference between amplitudes of different order in the electric field of the pulse (e.g., between the first and second orders in the case of the process investigated in Ref. [12]). 
In this paper we present a perturbative treatment of the problem of ionization (or detachment) of an atomic system by a few-cycle attosecond pulse, which allows a more complete description of the process than is possible in the strongfield nonperturbative regime. In Sec. II we present formal expressions for the first- and second-order (in the pulse electric field) transition amplitudes and express the ionization probability in terms of the CEP of the pulse, showing the connection of our results to the nonperturbative treatment of Ref. [24]. General expressions for the doubly differential ionization probability are given in terms of the physical vectors of the problem, i.e., the pulse polarization, e, and the ionized electron momentum direction, $\hat{\mathbf{p}}$. Special cases of this general result are given for each of the three major pulse polarizations (i.e., elliptical, circular, and linear). In terms of a few dynamical parameters, these formulas exhibit the explicit dependence of the ionization probability on the CEP and the ionized electron's momentum direction. Moreover, for the cases of elliptical or circular pulse polarization, our formulas predict two kinds of dichroic effects. Adopting a single active electron model for the atom and restricting our analysis to initial $s$ or $p$ states, in Sec. III we then derive explicit quantum expressions for the dynamical parameters introduced in Sec. II.

In Sec. IV we present numerical results of our various formulas for the special case of an initial $s$ electron. For the $\mathrm{H}$ atom and the case of a linearly polarized pulse we compare the results of our perturbative treatment to results of Peng et al. [12] obtained by direct numerical solution of the time-dependent Schrödinger equation; agreement is shown to be excellent. We compare results for two different pulse envelope shapes, finding little significant difference between them. Asymmetries for electrons ionized from a Coulomb potential are compared to those for an electron detached from a zero-range potential (ZRP). We then present a variety of results on the asymmetries in the ionized electron angular distributions for different pulse polarizations, intensities, and CEPs.

Finally, in Appendixes A and B, we give explicit analytic formulas for the dynamical parameters in our general formulas for initial $s$ states for the cases of a ZRP and a Coulomb potential. In Appendix C, we analyze the contribution of certain photon emission processes to the doubly differential ionization probability.

\section{GENERAL PARAMETRIZATION OF THE IONIZATION PROBABILITY}

We consider the electric-dipole interaction of an atom with a few-cycle pulse having a time envelope $F_{0}(t)$, carrier frequency $\omega$, full width at half maximum (FWHM) $\tau$, CEP $\phi$, and a fixed time-independent polarization described by the polarization vector $\mathbf{e}$. In general, the vector $\mathbf{e}$ is complex $\left(\mathbf{e} \cdot \mathbf{e}^{*}=1\right)$ and may be parameterized as for the case of a monochromatic light wave,

$$
\mathbf{e}=(\hat{\boldsymbol{\epsilon}}+i \eta \hat{\zeta}) / \sqrt{1+\eta^{2}},
$$

where $-1 \leq \eta \leq 1, \hat{\boldsymbol{\zeta}}=[\hat{\mathbf{k}} \times \hat{\boldsymbol{\epsilon}}]$, and $\hat{\mathbf{k}}, \hat{\boldsymbol{\epsilon}}$, and $\hat{\boldsymbol{\zeta}}$ indicate, respectively, the propagation direction of the pulse and the major and minor axes of the polarization ellipse. For later use, we define also the degrees of linear and circular polarization of the pulse, $\ell$ and $\xi$ :

$$
\begin{gathered}
\ell \equiv\left(1-\eta^{2}\right) /\left(1+\eta^{2}\right)=(\mathbf{e} \cdot \mathbf{e}) \\
\xi \equiv 2 \eta /\left(1+\eta^{2}\right)=i\left(\hat{\mathbf{k}} \cdot\left[\mathbf{e} \times \mathbf{e}^{*}\right]\right) \\
\ell^{2}+\xi^{2}=1 .
\end{gathered}
$$

(Thus, e.g., linear polarization corresponds to $\eta=0$ or $\ell=1$ and $\xi=0$; right and left circular polarization corresponds to $\eta= \pm 1$ or $\ell=0$ and $\xi= \pm 1$.) The electric field of the pulse may thus be expressed as follows:

$$
\mathbf{F}(t)=F_{0}(t) \operatorname{Re}\left[\mathbf{e} e^{-i(\omega t+\phi)}\right]=\frac{1}{2} F_{0}(t)\left[\mathbf{e} e^{-i(\omega t+\phi)}+\mathbf{e}^{*} e^{i(\omega t+\phi)}\right],
$$

and its Fourier transform is defined as $\hat{\mathbf{F}}(\varepsilon)$, i.e.,

$$
\hat{\mathbf{F}}(\varepsilon)=\int_{-\infty}^{\infty} \mathbf{F}(t) e^{i \varepsilon t} d t
$$

This Fourier transform comprises two terms,

$$
\hat{\mathbf{F}}(\varepsilon)=\mathbf{e}_{\phi} \hat{F}^{+}(\varepsilon)+\mathbf{e}_{\phi}^{*} \hat{F}^{-}(\varepsilon),
$$

where the factors $\hat{F}^{ \pm}(\varepsilon)$ are the CEP- and polarizationindependent scalar parameters of the pulse,

$$
\hat{F}^{ \pm}(\varepsilon)=\frac{1}{2} \int F_{0}(t) e^{i(\varepsilon \bar{\mp} \omega) t} d t, \quad \hat{F}^{-}(\varepsilon)=\left[\hat{F}^{+}(-\varepsilon)\right]^{*},
$$

and the single complex unit vector $\mathbf{e}_{\phi}\left(\mathbf{e}_{\phi} \cdot \mathbf{e}_{\phi}^{*}=1\right)$ contains the dependence on the CEP and the polarization:

$$
\mathbf{e}_{\phi}=\mathbf{e} e^{-i \phi} \text {. }
$$

Assuming that the strength of the pulse electric field is sufficiently weak so that time-dependent PT in powers of the pulse electric field may be employed, our goal is to parametrize the differential probability for ionization of a single electron with momentum $\mathbf{p}$ into the solid angle $d \Omega$ and the energy interval $d E$,

$$
\frac{d^{2} W}{d \Omega d E} \equiv \mathcal{W}
$$

where $E=p^{2} /(2 m)$. Within the PT approach, the dependence of $\mathcal{W}$ on the CEP, the pulse polarization, and the ejected electron direction $\hat{\mathbf{p}}$ can be parametrized $a b$ initio using rotational invariance and symmetry arguments without specification of a particular atom (cf. similar analyses for ionization by a monochromatic light wave in Refs. [25,26]). For simplicity, we assume the target atom to be randomly oriented (i.e., unpolarized and unaligned) and that neither the spin state of the electron nor the angular momentum state of the residual ion are detected (though these restrictions are not essential, cf. Ref. [25]). In this case, the problem involves only two vectors: the electron momentum unit vector $\hat{\mathbf{p}}$ and the complex vector $\mathbf{e}_{\phi}$ that is responsible for the polarization 
and CEP effects, while the atomic properties are described by true scalar parameters. Note that the problem also involves a pseudoscalar quantity, the degree of circular polarization $\xi$ (see, e.g., Ref. [27]).

The parametrization of the CEP dependence of $\mathcal{W}$ follows from very general considerations concerning the ionization amplitude, $A_{N}$, which is $N$ th order in the electric dipole interaction, $\hat{V}=-\hat{\mathbf{D}} \cdot \mathbf{F}(t)$, where $\hat{\mathbf{D}}$ is the dipole moment of the atom. Using Eq. (5), $\hat{V}$ has the form

$$
\hat{V}=-\frac{1}{2} F_{0}(t)\left(\mathbf{e}_{\phi} \cdot \hat{\mathbf{D}} e^{-i \omega t}+\mathbf{e}_{\phi}^{*} \cdot \hat{\mathbf{D}} e^{i \omega t}\right) .
$$

The amplitude $A_{N}$ is thus an $N$-linear form in the vectors $\mathbf{e}_{\phi}$ and $\mathbf{e}_{\phi}^{*}$, i.e., each interaction $\hat{V}$ introduces into the amplitude $A_{N}$ the phase factors $e^{-i \phi}$ and $e^{i \phi}$. Therefore, $\mathcal{W}$ has the form of a linear combination of terms involving $\cos (n \phi)$ and $\sin (n \phi)$ with $n \leq 2 N$. Moreover, the terms $\cos (n \phi)$ and $\sin (n \phi)$ have opposite parities under time inversion $(t \rightarrow-t)$ since the phase $\phi$ is a $t$-odd quantity [cf. Eq. (5)]. Therefore, in order that all terms in $\mathcal{W}$ have the same temporal symmetry, the factors multiplying the terms in $\mathcal{W}$ proportional to $\sin (n \phi)$ should also be $t$ odd, which will occur if these factors involve an interference between the real and imaginary (i.e., non-Hermitian) parts of different constituents of the ionization amplitude (cf. Refs. [25,26]). From these considerations, we conclude that in the nonperturbative regime (in which all orders of PT contribute to the ionization amplitude) the general expression for $\mathcal{W}$ can be presented as a Fourier series in the CEP $\phi$, in agreement with the nonperturbative quantum-mechanical analysis in Ref. [24] for a linearly polarized few-cycle pulse. (Note that our analysis above for the CEP dependence of $\mathcal{W}$ is general and is not limited to the case of a randomly oriented target.)

The carrier wave frequency of a few-cycle pulse determines the complexity of any parametrization of the ionization probability $\mathcal{W}$ of an atom. For the case of an ellipticallypolarized few-cycle pulse having a carrier frequency in the optical regime, the polarization-angular structure of the PT result for $\mathcal{W}$ is cumbersome because even the lowest-order ionization amplitude is of high order in the field $\mathbf{F}(t)$. For the case of few-cycle xuv pulses, however, in which the carrier frequency $\omega$ is such that $\left(E_{n}+\hbar \omega\right)>0$ (where $E_{n}$ is the energy of an outer electron of the atom), ionization is possible already in first-order PT in the pulse-atom interaction $\hat{V}$. A general parametrization of the first-order PT result for $\mathcal{W}$, $\mathcal{W}_{1}$, follows from the general form (11) of the operator $\hat{V}$ : it can involve only pairs of the four vectors, $\mathbf{e}_{\phi}, \mathbf{e}_{\phi}, \mathbf{e}_{\phi}^{*}$, and $\mathbf{e}_{\phi}^{*}$, which should form scalar products either with each other or with the vector $\hat{\mathbf{p}}$. Only four independent scalar products can be composed from these vectors:

$$
\begin{gathered}
\mathbf{e}_{\phi} \cdot \mathbf{e}_{\phi}^{*}=1, \quad\left|\mathbf{e}_{\phi} \cdot \hat{\mathbf{p}}\right|^{2}=|\mathbf{e} \cdot \hat{\mathbf{p}}|^{2}, \\
\mathbf{e}_{\phi} \cdot \mathbf{e}_{\phi}=\ell e^{-2 i \phi}, \quad\left(\mathbf{e}_{\phi} \cdot \hat{\mathbf{p}}\right)^{2}=(\mathbf{e} \cdot \hat{\mathbf{p}})^{2} e^{-2 i \phi},
\end{gathered}
$$

where the combinations [Eq. (12b)] are complex and thus equivalent to four real terms. Thus, the general parametrization for $\mathcal{W}_{1}$ has the following invariant form:

$$
\mathcal{W}_{1}=\mathcal{C}\left\{1+\beta_{0}\left(3|\mathbf{e} \cdot \hat{\mathbf{p}}|^{2}-1\right) / 2+\operatorname{Re}\left\{e^{-2 i \phi}\left[\beta_{1} \ell+\beta_{2}(\mathbf{e} \cdot \hat{\mathbf{p}})^{2}\right]\right\}\right\},
$$

involving six scalar parameters $\left(\mathcal{C}, \beta_{0}, \operatorname{Re} \beta_{1}, \operatorname{Im} \beta_{1}, \operatorname{Re} \beta_{2}\right.$, and $\operatorname{Im} \beta_{2}$ ) that depend only on the pulse shape and the atomic dynamics, while the entire dependence on the CEP $\phi$, the pulse polarization $\mathbf{e}$, and the ionized electron direction $\hat{\mathbf{p}}$ have been extracted explicitly. The first two terms in the parametrization [Eq. (13)] have the form of the usual differential cross section for photoionization of a randomly oriented target by a monochromatic field, in which case $4 \pi \mathcal{C}$ is the total photoionization cross section and $\beta_{0}$ is the "asymmetry parameter" (see, e.g., Refs. [25,28,29]). The other two terms in Eq. (13) are nonzero only for a short pulse as they originate from the interference of the amplitudes for two ionization pathways to the same continuum state of energy $E$. Specifically, they correspond to the contribution of the product of the scalar parameters $\hat{F}^{+}$and $\hat{F}^{-}$in Eq. (7) to the first-order ionization probability. Since each of these parameters in the operator $\hat{V}$ is accompanied by one of the phase factors $\left(e^{-i \phi}\right.$ or $\left.e^{i \phi}\right)$, the CEP dependence of their interference is obvious. Note that in the case of $L S$-coupling (in which the orbital and spin angular momenta are separately conserved) the number of atom-specific scalar parameters in Eq. (13) reduces to three $\left(\mathcal{C}, \operatorname{Re} \beta_{2}, \operatorname{Im} \beta_{2}\right)$ when the total orbital angular momenta of both the target atom $\left(L_{0}\right)$ and the residual ion $\left(L_{i}\right)$ are zero. Indeed, in this case the transition amplitude $A_{N}$ in any PT order is a scalar (instead of the direct product of spherical tensors with ranks $L_{0}$ and $L_{i}$ in the general case) so that an invariant parametrization in terms of the vectors of the problem exists directly for $A_{1}$,

$$
A_{1}=\alpha_{+}\left(\mathbf{e}_{\phi} \cdot \hat{\mathbf{p}}\right)+\alpha_{-}\left(\mathbf{e}_{\phi}^{*} \cdot \hat{\mathbf{p}}\right) .
$$

For this case, Eq. (13) for $\mathcal{W}_{1} \propto\left|A_{1}\right|^{2}$ simplifies, since $\beta_{0}=2$ and $\beta_{1}=0$ (cf. Ref. [30]). Note finally that Eq. (13) shows that the angular distribution in the lowest PT order is symmetric with respect to the substitution $\hat{\mathbf{p}} \rightarrow-\hat{\mathbf{p}}$. An asymmetry only appears in the high-order PT corrections to $\mathcal{W}_{1}$, which we consider next.

The general analysis of higher-order PT corrections to $\mathcal{W}_{1}$ leads to cumbersome expressions for $\mathcal{W}$, for which reason we carry out this analysis by making three simplifying assumptions: (i) we assume that the pulse bandwidth is less than $\left|E_{n}-\hbar \omega\right|$ (i.e., we exclude extremely short pulses) so that the first-order ionization amplitude is dominated by the "positive frequency" component $\left(\hat{F}^{+}\right)$of the Fouriertransform $\hat{\mathbf{F}}(\varepsilon)$ in Eq. (7); (ii) as follows from assumption (i), in the second-order PT amplitude $A_{2}$ we neglect terms involving two successive "negative frequency" $\left(\hat{F}^{-}\right)$components of $\hat{\mathbf{F}}(\varepsilon)$; (iii) we restrict our considerations to only the next-order (in $\hat{V}$ ) correction to the lowest-order PT result for $\mathcal{W}$, i.e., we neglect the absolute square of the second-order PT amplitude $A_{2}$ and take into account only its interference with the first-order amplitude $A_{1}$. [In Sec. IV below, our numerical results for a two-cycle $(\approx 100$ as) pulse with $\hbar \omega$ $\gtrsim 36 \mathrm{eV}$ show that these three assumptions are well justified up to a peak pulse intensity of the order of $10^{16} \mathrm{~W} / \mathrm{cm}^{2}$.] 


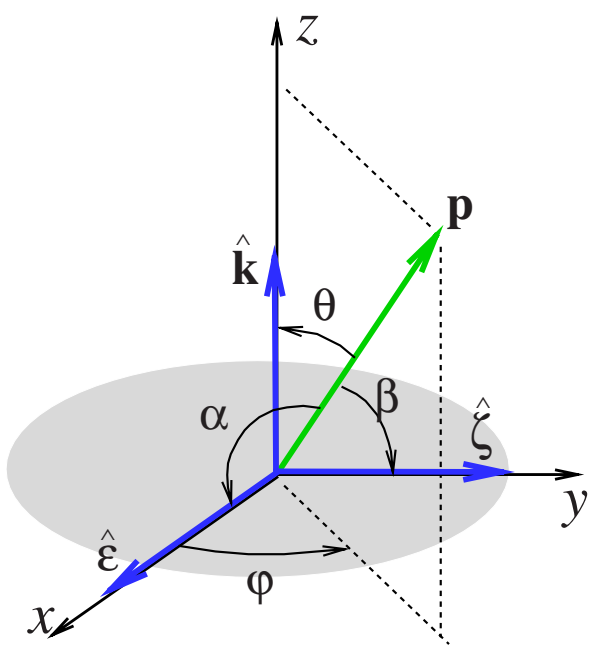

FIG. 1. (Color online) The physical vectors of the problem: the electron momentum $\mathbf{p}$, the major $(\hat{\boldsymbol{\epsilon}})$ and minor $(\hat{\boldsymbol{\zeta}})$ axes of the polarization ellipse, and the propagation direction $\hat{\mathbf{k}}$ of the pulse, as well as the angles between them.

To parametrize the interference $\left[\sim \operatorname{Re}\left(A_{1}^{*} A_{2}\right)\right]$ of the firstand second-order ionization amplitudes, we note that $A_{1}^{*}$ $\sim \mathbf{e}_{\phi}^{*}$ [according to assumption (i)], while $A_{2}$ involves two pairs of vectors $\mathbf{e}_{\phi}: \mathbf{e}_{\phi}, \mathbf{e}_{\phi}$ and $\mathbf{e}_{\phi}, \mathbf{e}_{\phi}^{*}$. [The pair $\mathbf{e}_{\phi}^{*}, \mathbf{e}_{\phi}^{*}$ does not contribute owing to assumption (ii).] Thus the interference term involves all independent scalar products composed from the electron momentum unit vector $\hat{\mathbf{p}}$ and the following two triads of pulse polarization vectors: $\mathbf{e}_{\phi}^{*}, \mathbf{e}_{\phi}, \mathbf{e}_{\phi}$ and $\mathbf{e}_{\phi}^{*}, \mathbf{e}_{\phi}, \mathbf{e}_{\phi}^{*}$. It is convenient to use the following three combinations of scalar products:

$$
\begin{gathered}
\left(\mathbf{e}_{\phi} \cdot \mathbf{e}_{\phi}^{*}\right)\left(\mathbf{e}_{\phi} \cdot \hat{\mathbf{p}}\right)=(\mathbf{e} \cdot \hat{\mathbf{p}}) e^{-i \phi}, \\
\left(\mathbf{e}_{\phi} \cdot \mathbf{e}_{\phi}\right)\left(\mathbf{e}_{\phi}^{*} \cdot \hat{\mathbf{p}}\right)=\ell\left(\mathbf{e}^{*} \cdot \hat{\mathbf{p}}\right) e^{-i \phi}, \\
\left(\mathbf{e}_{\phi} \cdot \hat{\mathbf{p}}\right)\left|\mathbf{e}_{\phi} \cdot \hat{\mathbf{p}}\right|^{2}=(\mathbf{e} \cdot \hat{\mathbf{p}})|\mathbf{e} \cdot \hat{\mathbf{p}}|^{2} e^{-i \phi} .
\end{gathered}
$$

As a result, the parametrization of $\mathcal{W}$ taking into account the lowest two orders of PT may be presented in two equivalent forms,

$$
\begin{aligned}
\mathcal{W} & =w_{1}+\operatorname{Re}\left\{e^{-i \phi}\left[\left(\gamma_{1}+\gamma_{2}|\mathbf{e} \cdot \hat{\mathbf{p}}|^{2}\right)(\mathbf{e} \cdot \hat{\mathbf{p}})+\gamma_{3} \ell\left(\mathbf{e}^{*} \cdot \hat{\mathbf{p}}\right)\right]\right\} \\
& =w_{1}+\operatorname{Re}\left\{(\mathbf{e} \cdot \hat{\mathbf{p}})\left[\left(\gamma_{1}+\gamma_{2}|\mathbf{e} \cdot \hat{\mathbf{p}}|^{2}\right) e^{-i \phi}+\gamma_{3}^{*} \ell e^{i \phi}\right]\right\},
\end{aligned}
$$

where

$$
w_{1}=\mathcal{C}\left[1+\beta_{0}\left(3|\mathbf{e} \cdot \hat{\mathbf{p}}|^{2}-1\right) / 2\right] .
$$

Three complex numbers $\gamma_{i}$ in Eqs. (16) comprise six real dynamical parameters that depend on the pulse shape and the atomic structure and may be expressed in terms of interference between radial parts of the first-order amplitude $A_{1}$ and different constituents of the second-order ionization amplitude $A_{2}$ (see Sec. III). Equations (16) make obvious two major results of our general phenomenological analysis: (i) For a given few-cycle xuv pulse [Eq. (5)], a measurement (or calculation) of the parameters $\gamma_{i}$ for a fixed electron energy $E$ and fixed CEP $\phi$ (say, $\phi=0$ ), allows one to reproduce the ionized electron angular distribution for any $\phi$ by using Eq. (16). (This result is consistent with corresponding results of nonperturbative quantum-mechanical analyses for the cases of linearly [24] and circularly [31] polarized fewcycle pulses.)

(ii) Equation (16b) shows that all asymmetries in the electron angular distributions are described by the scalar product $(\mathbf{e} \cdot \hat{\mathbf{p}})$, which changes sign when $\mathbf{p} \rightarrow-\mathbf{p}$ and which depends on the pulse polarization. As is clear from our parametrization [Eq. (1)] for $\mathbf{e},(\mathbf{e} \cdot \hat{\mathbf{p}})$ is proportional to $\sin \theta$ (where $\theta$ is the angle between $\mathbf{p}$ and $\hat{\mathbf{k}}$ ). Thus these asymmetries vanish in the direction of the pulse propagation and achieve their maximum in the polarization plane $(\theta=\pi / 2)$.

\section{A. Ionization probability for an elliptically polarized pulse}

By writing the scalar product $(\mathbf{e} \cdot \hat{\mathbf{p}})$ in the form

$$
(\mathbf{e} \cdot \hat{\mathbf{p}})=\frac{\cos \alpha+i \eta \cos \beta}{\sqrt{1+\eta^{2}}}=\frac{(1+\ell) \cos \alpha+i \xi \cos \beta}{\sqrt{2(1+\ell)}},
$$

the explicit angular and polarization dependence of $\mathcal{W}$ in Eqs. (16) may be presented in the most compact form in terms of the angles $\alpha$ and $\beta$ between the electron momentum $\mathbf{p}$ and the major $(\hat{\boldsymbol{\epsilon}})$ and minor $(\hat{\zeta})$ axes of the polarization ellipse, respectively (cf. Fig. 1). Using also the related expression, obvious from Eq. (18),

$$
2|\mathbf{e} \cdot \hat{\mathbf{p}}|^{2}=(1+\ell) \cos ^{2} \alpha+(1-\ell) \cos ^{2} \beta,
$$

Eq. (16a) takes the following form:

$$
\begin{aligned}
\mathcal{W}= & w_{1}+\frac{1}{\sqrt{2(1+\ell)}}\left[( 1 + \ell ) \operatorname { c o s } \alpha \operatorname { R e } \left\{\left(\gamma_{1}+\gamma_{2}|\mathbf{e} \cdot \hat{\mathbf{p}}|^{2}\right.\right.\right. \\
& \left.\left.\left.+\gamma_{3} \ell\right) e^{-i \phi}\right\}-\xi \cos \beta \operatorname{Im}\left\{\left(\gamma_{1}+\gamma_{2}|\mathbf{e} \cdot \hat{\mathbf{p}}|^{2}-\gamma_{3} \ell\right) e^{-i \phi}\right\}\right],
\end{aligned}
$$

where the polarization and angular dependence of the lowestorder term $w_{1}$ [cf. Eq. (17)] follows from Eq. (19).

An alternative to the parametrization of $\mathcal{W}$ in Eq. (20) in terms of the spherical coordinate angles, $\theta$ and $\varphi$, of the vector $\mathbf{p}$ [in a reference frame with $z$ axis along the pulse propagation direction $\hat{\mathbf{k}}$ and $x$ axis along $\hat{\boldsymbol{\epsilon}}$ (cf. Fig. 1)] is sometimes useful. In this case, $\mathcal{W}$ has form (20) with the substitutions $\cos \alpha=\sin \theta \cos \varphi \quad$ and $\quad \cos \beta=\sin \theta \sin \varphi$, while Eq. (19) transforms to

$$
2|\mathbf{e} \cdot \hat{\mathbf{p}}|^{2}=\sin ^{2} \theta(1+\ell \cos 2 \varphi) .
$$

These two alternative parametrizations simplify and become equal in the polarization plane $(\theta=\pi / 2)$, in which case $\beta$ $=\pi / 2-\alpha$ and $\alpha=\varphi$ : 


$$
\begin{aligned}
\mathcal{W}= & \mathcal{C}\left[1+\beta_{0}(1+3 \ell \cos 2 \varphi) / 4\right]+\frac{1}{\sqrt{2(1+\ell)}}[(1+\ell) \\
& \times \cos \varphi \operatorname{Re}\left\{\left[\gamma_{1}+\gamma_{2}(1+\ell \cos 2 \varphi) / 2+\gamma_{3} \ell\right] e^{-i \phi}\right\} \\
& \left.-\xi \sin \varphi \operatorname{Im}\left\{\left[\gamma_{1}+\gamma_{2}(1+\ell \cos 2 \varphi) / 2-\gamma_{3} \ell\right] e^{-i \phi}\right\}\right]
\end{aligned}
$$

\section{B. Circular and elliptic dichroism effects}

Equation (20) gives the explicit dependence of the electron angular distribution on the degrees of circular and linear polarization, $\xi$ and $l$, of an elliptically polarized few-cycle pulse. This dependence has the following form:

$$
\mathcal{W}=\delta_{0}+\xi \delta_{C D}+\xi \ell \delta_{E D},
$$

where $\delta_{0}$ is invariant under a change in sign of $\xi$ (i.e., under the transformation $\mathbf{e} \rightarrow \mathbf{e}^{*}$ ), while the other two terms are dichroic (i.e., they change sign when $\xi \rightarrow-\xi$ ). The term proportional to $\xi$ represents the circular dichroism (CD) effect, while the term proportional to $\xi \ell$ describes the elliptic dichroism (ED) effect, which (unlike the CD effect) vanishes for purely circular polarization, i.e., $\xi= \pm 1$ and $\ell=0$. The parameters $\delta_{0}, \delta_{C D}$, and $\delta_{E D}$ depend on the dynamical parameters $\gamma_{i}$ defined in Eq. (16a) and the CEP $\phi$ via the real and imaginary parts of $\gamma_{i} e^{-i \phi}$, as follows:

$$
\begin{gathered}
\delta_{0}=w_{1}+\sqrt{(1+\ell) / 2} \cos \alpha \operatorname{Re}\left\{\left(\gamma_{1}+\gamma_{2}|\mathbf{e} \cdot \hat{\mathbf{p}}|^{2}+\gamma_{3} \ell\right) e^{-i \phi}\right\}, \\
\delta_{C D}=-\frac{1}{\sqrt{2(1+\ell)}} \cos \beta \operatorname{Im}\left\{\left(\gamma_{1}+\gamma_{2}|\mathbf{e} \cdot \hat{\mathbf{p}}|^{2}\right) e^{-i \phi}\right\}, \\
\delta_{E D}=\frac{1}{\sqrt{2(1+\ell)}} \cos \beta \operatorname{Im}\left\{\gamma_{3} e^{-i \phi}\right\} .
\end{gathered}
$$

Both $\mathrm{CD}$ and $\mathrm{ED}$ effects are maximal for $\cos \beta=1$, i.e., when the electron momentum $\mathbf{p}$ lies in the polarization plane along the minor axis of the polarization ellipse.

The terms $\sim \xi$ and $\sim \xi \ell$ in Eq. (23) describe the difference in the ionized electron angular distributions for opposite helicities of the elliptically polarized pulse field $\mathbf{F}(t)$. This difference is counterintuitive since it is well known that dichroic effects do not occur in ordinary photoionization of a randomly oriented atomic target. In the present case, however, these effects originate from the strong-field corrections to the first-order ionization amplitude and so should not be surprising. (For a brief review of dichroic effects in photoprocesses with unpolarized atomic targets, see Ref. [32]). Nevertheless, there is a significant difference between dichroic effects for monochromatic and few-cycle pulses. In the former case, the dichroic effects have a purely dynamical origin ("dynamical dichroism") since they originate from an interference between the real and imaginary parts of (generally, non-Hermitian) transition amplitudes (cf. Ref. [32] for details). This non-Hermiticity of the transition amplitudes is the reason that our phenomenological parameters $\gamma_{i}$ are complex. Thus the "dynamical" parts of the CD and ED param- eters in our case are given, respectively, by the terms in Eqs. (25) and (26) that are proportional to $\cos \phi$. However, there are the additional "kinematical" (or CEP-induced) parts of the parameters $\delta_{C D}$ and $\delta_{E D}$ that are proportional to $\sin \phi$ and involve the real parts of the dynamical atomic parameters $\gamma_{i}$. Based on these considerations, we can conclude that fewcycle pulse calculations using the standard strong-field approximation (in which case the transition amplitudes are Hermitian) can only reproduce the CEP-induced part of dichroic effects in strong-field photoprocesses.

\section{Ionization probability for a linearly polarized pulse}

The general parametrization [Eq. (20)] simplifies significantly for the cases of linear $(\xi=0)$ and circular $(\ell=0)$ polarizations. In the former case, one has $(\mathbf{e} \cdot \hat{\mathbf{p}})=\cos \alpha$ so that the angular distribution depends only on the angle $\alpha$ between the polarization axis and the ionized electron momentum direction. Using Eqs. (19) and (20), the ionization probability in this case becomes

$$
\begin{aligned}
\mathcal{W}= & \mathcal{C}\left[1+\beta_{0}\left(3 \cos ^{2} \alpha-1\right) / 2\right] \\
& +\cos \alpha \operatorname{Re}\left\{\left(\gamma_{1}+\gamma_{2} \cos ^{2} \alpha+\gamma_{3}\right) e^{-i \phi}\right\} .
\end{aligned}
$$

The asymmetry in the ionized electron angular distribution stems only from the interference term in Eq. (27), which is odd in $\cos \alpha$. The asymmetry is maximal along the polarization axis and may be written as follows:

$$
\begin{aligned}
\left.\frac{d \Delta}{d E} \equiv \mathcal{W}\right|_{\alpha=0}-\left.\mathcal{W}\right|_{\alpha=\pi} & =2 \operatorname{Re}\left[\left(\gamma_{1}+\gamma_{2}+\gamma_{3}\right) e^{-i \phi}\right] \\
& =2\left|\gamma_{1}+\gamma_{2}+\gamma_{3}\right| \cos \left(\phi-\Theta_{L}\right),
\end{aligned}
$$

where $\Theta_{L} \equiv \arg \left(\gamma_{1}+\gamma_{2}+\gamma_{3}\right)$. We emphasize that, as for dynamic dichroism effects, the "shift angle" $\Theta_{L}$ vanishes for the case of real parameters $\gamma_{i}$.

\section{Ionization probability for a circularly polarized pulse}

For the case of circular polarization, it is convenient to use the spherical coordinate angles $\theta, \varphi$ to describe the angular distribution since for $\ell=0$ Eq. (21) gives

$$
2|\mathbf{e} \cdot \hat{\mathbf{p}}|^{2}=\sin ^{2} \theta,
$$

while $(\mathbf{e} \cdot \hat{\mathbf{p}})$ may be presented as follows:

$$
(\mathbf{e} \cdot \hat{\mathbf{p}})=|\mathbf{e} \cdot \hat{\mathbf{p}}| e^{i \xi \varphi}=(1 / \sqrt{2}) \sin \theta e^{i \xi \varphi}, \quad \xi= \pm 1 .
$$

As a result, we have

$$
\begin{aligned}
\mathcal{W}= & \mathcal{C}\left[1-\beta_{0}\left(3 \cos ^{2} \theta-1\right) / 4\right]+(1 / \sqrt{2}) \\
& \times \sin \theta \operatorname{Re}\left\{\left[\gamma_{1}+(1 / 2) \gamma_{2} \sin ^{2} \theta\right] e^{i(\xi \varphi-\phi)}\right\}
\end{aligned}
$$

or in the polarization plane $(\theta=\pi / 2)$,

$$
\mathcal{W}=\mathcal{C}\left(1+\beta_{0} / 4\right)+(1 / \sqrt{2})\left|\gamma_{1}+\gamma_{2} / 2\right| \cos \left(\xi \varphi-\phi+\Theta_{C}\right)
$$

where 


$$
\Theta_{C} \equiv \arg \left(\gamma_{1}+\gamma_{2} / 2\right) .
$$

[Note that Eq. (32) may be presented in a form similar to Eq. (33) but with a $\theta$-dependent "shift angle" $\Theta_{C}(\theta)$.] Using Eq. (33) and the relations $\cos (\xi \varphi)=\cos \varphi$ and $\sin (\xi \varphi)=\xi \sin \varphi$, the CD parameter [Eq. (25)] for purely circular polarization may be written in the polarization plane in the following form:

$$
\delta_{C D}=(1 / \sqrt{2})\left|\gamma_{1}+\gamma_{2} / 2\right| \sin \varphi\left(\sin \phi \cos \Theta_{C}-\sin \Theta_{C} \cos \phi\right),
$$

showing explicitly the dynamical $\left(\sim \sin \Theta_{C}\right)$ and CEPinduced $(\sim \sin \phi)$ contributions to the parameter $\delta_{C D}$.

A remarkable feature of Eqs. (32) and (33) is that in both cases the angular distributions depend on the angle $\varphi$, the CEP $\phi$, and the sign of $\xi(= \pm 1)$ only through the combination $\xi \varphi-\phi$. This fact is a consequence of the symmetry of the TDSE in a circularly polarized pulse [31] so that changing the CEP $\phi$ to $\phi+\Delta \phi$ is equivalent to a rotation of the three-dimensional angular distribution around the direction of pulse propagation by the angle $\Delta \phi$, counterclockwise for right circularly polarized light $(\xi=1)$ or clockwise for left circularly polarized light $(\xi=-1)$ (cf. Ref. [31]). The CEP $\phi$ thus has no absolute meaning in the case of a circularly polarized pulse, i.e., its value depends on the choice of the laboratory coordinate system. Indeed, in this case the major axis of the polarization ellipse $\hat{\boldsymbol{\epsilon}}$ can be chosen arbitrarily. Nevertheless, the asymmetry in the ionized electron angular distribution stems from the asymmetry of the few-cycle pulse. (Since the target atoms are randomly oriented, there is no preferred direction in the atomic medium.) Thus, the CEP in the case of a circularly polarized pulse may be characterized by means of the azimuthal angle at which the pulse reaches its maximum magnitude (in a fixed laboratory coordinate system with $z$ axis along the vector $\hat{\mathbf{k}}$ ). For example, if we choose $\hat{\boldsymbol{\epsilon}}$ along the $x$ axis and assume that the pulse reaches its maximum magnitude at $t=0$, then according to Eq. (5) the components of the pulse electric field are given by $F_{x}(0)=\frac{1}{\sqrt{2}} F_{0}(0) \cos \phi$ and $F_{y}(0)= \pm \frac{1}{\sqrt{2}} F_{0}(0) \sin \phi$. Hence, for a pulse having a degree of circular polarization $\xi= \pm 1$, the pulse electric field reaches its maximum value $\frac{1}{\sqrt{2}} F_{0}(0)$ at the angle $\varphi= \pm \phi$.

\section{ANALYTICAL EXPRESSIONS FOR THE DYNAMICAL PARAMETERS}

To derive explicit expressions for the dynamical parameters $\mathcal{C}, \beta_{i}$, and $\gamma_{i}$ introduced in the previous section, we begin from the quantum mechanical expression for $\mathcal{W}$ in Eq. (10) within a perturbative approach (in the pulse electric field). For simplicity, we adopt the single active electron approximation and assume that $\psi_{n l m}$ describes the initial bound state of the electron with energy $E_{n}$ and that $\psi_{\mathbf{p}}^{(-)}$describes a final continuum state with electron momentum $\mathbf{p}$ and energy $E=p^{2} / 2$, both of which are solutions of the time-independent Schrödinger equation for an electron in a potential $U(r)$ (atomic units are employed in the rest of this paper, unless otherwise noted):

$$
\begin{aligned}
& {\left[-\nabla^{2} / 2+U(r)\right] \psi_{n l m}(\mathbf{r})=E_{n} \psi_{n l m}(\mathbf{r}),} \\
& {\left[-\nabla^{2} / 2+U(r)\right] \psi_{\mathbf{p}}^{(-)}(\mathbf{r})=\frac{p^{2}}{2} \psi_{\mathbf{p}}^{(-)}(\mathbf{r}) .}
\end{aligned}
$$

The ionization probability for an ionized electron ejected in the direction $\Omega$ with energy $E=p^{2} / 2$ is given by

$$
\begin{aligned}
\mathcal{W} & =\frac{p}{2 l+1} \sum_{m}\left|A_{1}+A_{2}+\cdots\right|^{2} \\
& \approx \frac{p}{2 l+1} \sum_{m}\left[\left|A_{1}\right|^{2}+2 \operatorname{Re}\left(A_{1}^{*} A_{2}\right)+\cdots\right] .
\end{aligned}
$$

Here $A_{1}$ and $A_{2}$ are the first and second-order transition amplitudes (in the electric dipole approximation):

$$
\begin{aligned}
A_{1} & =\left\langle\psi_{\mathbf{p}}^{(-)}\left|\hat{\mathbf{F}}\left(E-E_{n}\right) \cdot \mathbf{r}\right| \psi_{n l m}\right\rangle, \\
A_{2}= & -\frac{1}{2 \pi} \int_{-\infty}^{\infty} d \varepsilon\left\langle\psi_{\mathbf{p}}^{(-)}\right| \hat{\mathbf{F}}(E-\varepsilon) \cdot \mathbf{r} \\
& \times G_{\varepsilon}\left(\mathbf{r}, \mathbf{r}^{\prime}\right) \hat{\mathbf{F}}\left(\varepsilon-E_{n}\right) \cdot \mathbf{r}^{\prime}\left|\psi_{n l m}\right\rangle,
\end{aligned}
$$

where $G_{\varepsilon}$ is a stationary Green's function for the electron:

$$
G_{\varepsilon}\left(\mathbf{r}, \mathbf{r}^{\prime}\right)=\sum_{k l^{\prime} m^{\prime}} \frac{\left|\psi_{k l^{\prime} m^{\prime}}\right\rangle\left\langle\psi_{k l^{\prime} m^{\prime}}\right|}{E_{k}-\varepsilon}+\int d \mathbf{p} \frac{\left|\psi_{\mathbf{p}}^{(-)}\right\rangle\left\langle\psi_{\mathbf{p}}^{(-)}\right|}{p^{2} / 2-\varepsilon-i 0} .
$$

The amplitude $A_{2}$ may also be expressed in terms of the nonstationary Green's function $\mathcal{G}\left(\mathbf{r}, \mathbf{r}^{\prime} ; t-t^{\prime}\right)$ :

$$
\begin{aligned}
A_{2}= & \int_{-\infty}^{\infty} d t e^{i E t} \int_{-\infty}^{t} d t^{\prime} e^{-i E_{n^{\prime}}} \\
& \times\left\langle\psi_{\mathbf{p}}^{(-)}\left|\mathbf{F}(t) \cdot \mathbf{r} \mathcal{G}\left(\mathbf{r}, \mathbf{r}^{\prime} ; t-t^{\prime}\right) \mathbf{F}\left(t^{\prime}\right) \cdot \mathbf{r}^{\prime}\right| \psi_{n l m}\right\rangle,
\end{aligned}
$$

where

$$
\mathcal{G}\left(\mathbf{r}, \mathbf{r}^{\prime} ; t-t^{\prime}\right)=-\frac{1}{2 \pi} \int d \mathcal{E} G_{\mathcal{E}}\left(\mathbf{r}, \mathbf{r}^{\prime}\right) e^{-i \mathcal{E}\left(t-t^{\prime}\right)}
$$

For a spherically symmetric potential $U(r)$, the initial and final states can be expanded in spherical harmonics,

$$
\begin{gathered}
\left|\psi_{n l m}\right\rangle=Y_{l m}(\hat{\mathbf{r}})|n l\rangle, \\
\left\langle\psi_{\mathbf{p}}^{(-)}\right|=\frac{1}{p} \sum_{l m}(-i)^{l} e^{i \delta_{l} Y_{l m}(\hat{\mathbf{p}}) Y_{l m}^{*}(\hat{\mathbf{r}})\langle p l|,}
\end{gathered}
$$

where $\delta_{l}$ is the $l$ th partial wave phase shift, $|n l\rangle$ and $\langle p l|$ are, respectively, the radial parts of the bound and continuum state wave functions [with the latter having momentum normalization, i.e., $\left.\left\langle p l \mid p^{\prime} l\right\rangle=\delta\left(p-p^{\prime}\right)\right]$, and $\hat{\mathbf{r}}$ and $\hat{\mathbf{p}}$ are, respectively, the unit vectors along the radial, $\mathbf{r}$, and electron momentum, p, directions. Substituting Eq. (43) into Eqs. (38)-(40) and following the procedure described in detail in $[25,26]$ one can extract analytically the dependence of $\mathcal{W}$ on 
the physical vectors of the problem for an arbitrary angular momentum, $l$, of the initial state. For simplicity, we consider in this paper only ionization from initial $s$ and $p$ states.

\section{A. Amplitudes for an initial $s$ state}

In this case it is possible to parametrize not only $\left|A_{1}\right|^{2}$ and $\operatorname{Re}\left(A_{1}^{*} A_{2}\right)$ but also the first- and second-order transition amplitudes $A_{1}$ and $A_{2}$ themselves [see text above Eq. (14)]:

$$
\begin{gathered}
A_{1}=\alpha_{+}(\mathbf{e} \cdot \hat{\mathbf{p}}) e^{-i \phi}+\alpha_{-}\left(\mathbf{e}^{*} \cdot \hat{\mathbf{p}}\right) e^{i \phi} \\
A_{2}=M_{0+2}^{+-}-3 M_{2}^{+-}|\mathbf{e} \cdot \hat{\mathbf{p}}|^{2}+\left[\ell M_{0+2}^{++}-3 M_{2}^{++}(\mathbf{e} \cdot \hat{\mathbf{p}})^{2}\right] e^{-2 i \phi} \\
+\left[\ell M_{0+2}^{--}-3 M_{2}^{--}\left(\mathbf{e}^{*} \cdot \hat{\mathbf{p}}\right)^{2}\right] e^{2 i \phi}
\end{gathered}
$$

where

$$
\begin{gathered}
\alpha_{ \pm}=\mathcal{A}_{p 1} \hat{F}^{ \pm}\left(E-E_{n}\right), \\
M_{l}^{++}=\int d \varepsilon \hat{F}^{+}(E-\varepsilon) \hat{F}^{+}\left(\varepsilon-E_{n}\right) \mathcal{M}_{p l}(\varepsilon), \\
M_{l}^{--}=\int d \varepsilon \hat{F}^{-}(E-\varepsilon) \hat{F}^{-}\left(\varepsilon-E_{n}\right) \mathcal{M}_{p l}(\varepsilon), \\
M_{l}^{+-}=\int d \varepsilon\left[\hat{F}^{+}(E-\varepsilon) \hat{F}^{-}\left(\varepsilon-E_{n}\right)\right. \\
\left.+\hat{F}^{-}(E-\varepsilon) \hat{F}^{+}\left(\varepsilon-E_{n}\right)\right] \mathcal{M}_{p l}(\varepsilon), \\
M_{0+2}^{ \pm \pm}=M_{0}^{ \pm \pm}+M_{2}^{ \pm \pm}, \quad M_{0+2}^{+-}=M_{0}^{+-}+M_{2}^{+-} .
\end{gathered}
$$

The parameters $\mathcal{A}_{p 1}$ and $\mathcal{M}_{p l}(\varepsilon)(l=0,2)$ depend on the particular atomic system considered. In general, they can be expressed in terms of radial matrix elements:

$$
\begin{gathered}
\mathcal{A}_{p 1}=-\frac{i e^{i \delta_{1}}}{\sqrt{4 \pi} p}\langle p 1|r| n 0\rangle, \\
\mathcal{M}_{p l}(\varepsilon)=-\frac{e^{i \delta_{l}}}{12 \pi^{3 / 2} p}\left\langle p l\left|r g_{1}\left(\varepsilon ; r, r^{\prime}\right) r^{\prime}\right| n 0\right\rangle .
\end{gathered}
$$

Here $g_{1}(\varepsilon)$ is the radial part of the Green's function for the atomic system:

$$
G_{E}\left(\mathbf{r}, \mathbf{r}^{\prime}\right)=\sum_{l m} g_{l}\left(E ; r, r^{\prime}\right) Y_{l m}(\hat{\mathbf{r}}) Y_{l m}^{*}\left(\hat{\mathbf{r}}^{\prime}\right)
$$

For the cases of a ZRP and a Coulomb potential, the atomic parameters $\mathcal{A}_{p 1}, \mathcal{M}_{p 0}(\varepsilon)$, and $\mathcal{M}_{p 2}(\varepsilon)$ can be calculated analytically, as discussed in Appendixes A and B, respectively. The numerical evaluation of the first-order amplitude $A_{1}$ is simple, but the numerical evaluation of the second-order amplitude $A_{2}$ is quite difficult, especially for the case of a Coulomb potential (owing mainly to the integration over the intermediate state energy $\varepsilon$ ). Nevertheless, the numerical procedure for our perturbative treatment is much faster and easier than would be the case for a direct numerical solution of the TDSE.
Taking into account the assumptions discussed in the previous section [i.e., (i) neglecting the "negative frequency" component $\left(\hat{F}^{-}\right)$in the first-order ionization amplitude and (ii) neglecting terms involving two negative frequency components $\left(\hat{F}^{-}\right)$in the second-order PT amplitude], one obtains

$$
\widetilde{A}_{1} \approx \alpha_{+}(\mathbf{e} \cdot \hat{\mathbf{p}}) e^{-i \phi}
$$

$$
\tilde{A}_{2} \approx M_{0+2}^{+-}-3 M_{2}^{+-}|\mathbf{e} \cdot \hat{\mathbf{p}}|^{2}+\left[\ell M_{0+2}^{++}-3 M_{2}^{++}(\mathbf{e} \cdot \hat{\mathbf{p}})^{2}\right] e^{-2 i \phi} .
$$

Substituting Eqs. (55) and (56) into Eq. (38) and comparing the result with Eqs. (16) and (17), we obtain the following expressions for the dynamical parameters in the latter equations:

$$
\begin{gathered}
\mathcal{C}=\frac{p}{3}\left|\alpha_{+}\right|^{2}, \quad \beta_{0}=2, \\
\gamma_{1}=2 p \alpha_{+} M_{0+2}^{+-*}, \\
\gamma_{2}=-6 p\left(\alpha_{+} M_{2}^{+-*}+\alpha_{+}^{*} M_{2}^{++}\right), \\
\gamma_{3}=2 p \alpha_{+}^{*} M_{0+2}^{++} .
\end{gathered}
$$

Although the approximate amplitudes in Eqs. (55) and (56) are quite accurate and much simpler for analytical work, we note that in all numerical calculations presented in this paper we have used the exact amplitudes in Eqs. (45) and (46). A detailed discussion of the significance of the terms $\alpha$ and $M_{i}^{--}$[which are neglected in the approximate Eqs. (55) and (56)] is given in Appendix C. In addition, we note that in our numerical calculations we have included also the term $\left|A_{2}\right|^{2}$ [cf. Eq. (38)]. Although $\left|A_{2}\right|^{2}$ is in general small compared to both $\left|A_{1}\right|^{2}$ and $2 \operatorname{Re}\left(A_{1}^{*} A_{2}\right)$ and is of the same order in the laser pulse intensity as the term $\operatorname{Re}\left(A_{1}^{*} A_{3}\right) \sim I^{2}$ (where $A_{3}$ is the third-order amplitude), we have kept this term in order to ensure positive values for the ionization probability, $\mathcal{W}$, which can otherwise take negative values for final-state energies at which $\left|A_{1}\right|^{2}$ is small (so that higher orders of PT are needed to describe ionization at such energies correctly). In Fig. 11 we illustrate for a particular case the magnitudes of not only the terms we do include in our analytic formulas but also those that we do not include, with the latter shown to be quite small. We can thus use the above approximate analytic formulas to analyze and interpret CEP effects in ionized electron angular distributions produced by few-cycle attosecond xuv pulses. Most important, of course, is the secondorder PT amplitude in Eq. (56) since its interference with the first-order amplitude is what gives rise to these CEP effects.

\section{B. Dynamical parameters for an Initial $p$ state}

For an initial $p$ state we obtain the following expressions for the dynamical parameters in Eqs. (16) and (17) (using the same assumptions and procedures as for an initial $s$ state):

$$
\mathcal{C}=\frac{1}{36 \pi p}\left(R_{0}^{2}+2 R_{2}^{2}\right)\left|\hat{F}^{+}\left(E-E_{n}\right)\right|^{2},
$$



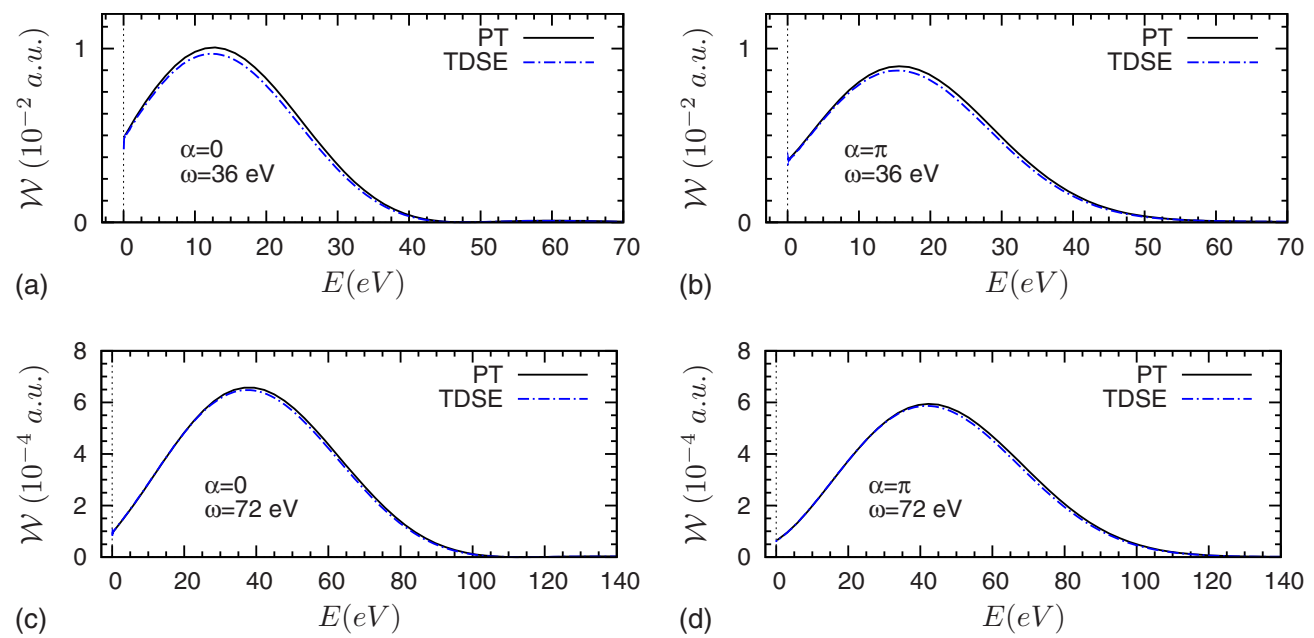

FIG. 2. (Color online) Comparison of the present PT results with TDSE results of Ref. [12] for the probability of ionization of the H atom by linearly polarized, two-cycle xuv pulses. Panels (a) and (b): pulse has carrier frequency $\omega=36 \mathrm{eV}$, CEP $\phi=0$, and peak intensity $I_{0}$ $=5 \times 10^{15} \mathrm{~W} / \mathrm{cm}^{2}$; panels $(\mathrm{c})$ and $(\mathrm{d})$ : pulse has carrier frequency $\omega=72 \mathrm{eV}, \mathrm{CEP} \phi=0$, and peak intensity $I_{0}=2 \times 10^{16} \mathrm{~W} / \mathrm{cm}^{2}$. Each pulse has a $\cos ^{2}$ envelope. Results in panels (a) and (c) are for ionization of electrons parallel to the polarization axis $(\alpha=0)$; those in panels (b) and (d) are for ionization of electrons antiparallel to the polarization axis $(\alpha=\pi)$.

$$
\beta_{0}=2 \frac{1-2\left(R_{0} / R_{2}\right) \cos \left(\delta_{0}-\delta_{2}\right)}{1+2\left(R_{0} / R_{2}\right)^{2}},
$$

where $R_{l}=\langle p l|r| n 1\rangle$.

The dynamical parameters for the interference term are

$$
\gamma_{1}=A^{++}+A^{-+*}+\left(B^{-+}+B^{+-}\right)^{*}+\left(C^{-+}+C^{+-}\right)^{*}+D^{+-*}+D^{++},
$$

$$
\begin{aligned}
& \gamma_{2}=-5\left[\left(C^{-+}+C^{+-}\right)^{*}+C^{++}\right], \\
& \gamma_{3}=A^{+-*}+B^{++}+C^{++}+D^{-+*},
\end{aligned}
$$

where

$$
\begin{aligned}
A^{\alpha \beta}= & \frac{1}{3}\left[10 \sqrt{7}\left(5 R_{10}^{0 \alpha \beta}-2 R_{10}^{2 \alpha \beta}\right)+5(4 \sqrt{7}+3) R_{12}^{0 \alpha \beta}\right. \\
& \left.-(26 \sqrt{7}-3) R_{12}^{2 \alpha \beta}+15 R_{30}^{2 \alpha \beta}+6(9 \sqrt{7}-2) R_{32}^{2 \alpha \beta}\right], \\
B^{\alpha \beta}= & 2 \sqrt{7}\left(5 R_{10}^{2 \alpha \beta}-5 R_{12}^{0 \alpha \beta}-11 R_{12}^{2 \alpha \beta}-6 R_{32}^{2 \alpha \beta}\right), \\
& C^{\alpha \beta}=5 R_{12}^{0 \alpha \beta}+R_{12}^{2 \alpha \beta}+5 R_{30}^{2 \alpha \beta}-4 R_{32}^{2 \alpha \beta}, \\
D^{\alpha \beta}= & 10 \sqrt{7} R_{10}^{2}-5(2 \sqrt{7}-1) R_{12}^{0}+(8 \sqrt{7}+1) R_{12}^{2}+5 R_{30}^{2} \\
& +2(9 \sqrt{7}-2) R_{32}^{2}, \\
R_{l l^{\prime}}^{L \alpha \beta}= & \frac{i}{600 \sqrt{21} \pi^{2} p} e^{i\left(\delta_{l^{-}} \delta_{l^{\prime}}\right)}\left\langle p l^{\prime}|r| n 1\right\rangle \hat{F}^{+}\left(E-E_{n}\right) \\
& \times \int d \varepsilon \hat{F}^{\alpha}(E-\varepsilon) \hat{F}^{\beta}\left(\varepsilon-E_{n}\right)\left\langle p l\left|r g_{L}\left(\varepsilon ; r, r^{\prime}\right) r^{\prime}\right| n 1\right\rangle .
\end{aligned}
$$

Since the CEP-dependent terms in Eq. (16) have the same form for initial $s$ and $p$ states, in Sec. IV we present numerical results only for an initial $s$ state.

\section{RESULTS AND DISCUSSION}

The above very general analysis for ionization of randomly oriented atoms by an attosecond xuv pulse (whose intensity is such that a PT analysis is appropriate and whose bandwidth is not too large) has shown that (i) interference of the first and second-order (in the pulse electric field) ionization amplitudes results in an asymmetry of the electron angular distribution and in dichroic effects; (ii) the CEP dependence of the ionization probability can be extracted analytically for arbitrary polarization of the pulse.

In this section we illustrate the predictions of our various PT formulas numerically. Specifically, we analyze the results for accuracy and compare our PT predictions for different atomic systems and for different pulse polarizations. Unless otherwise noted, our PT predictions are obtained by evaluating the ionization probability in Eq. (38) using the amplitudes defined in Eqs. (45)-(51).

\section{A. Comparison with TDSE results for the $\mathrm{H}$ atom}

In Fig. 2 we compare our PT predictions for the asymmetries in the ionized electron angular distribution for the hydrogen atom with results of a numerical solution of the TDSE [12]. Both results are for ionization by few-cycle xuv pulses that are linearly polarized along the $z$ axis and have a $\cos ^{2}$ envelope, i.e.,

$$
\mathbf{A}(t)=A_{0}(t) \mathbf{e}_{z} \cos (\omega t+\phi), \quad \mathbf{F}(t)=-\frac{1}{c} \frac{\partial \mathbf{A}(t)}{\partial t},
$$



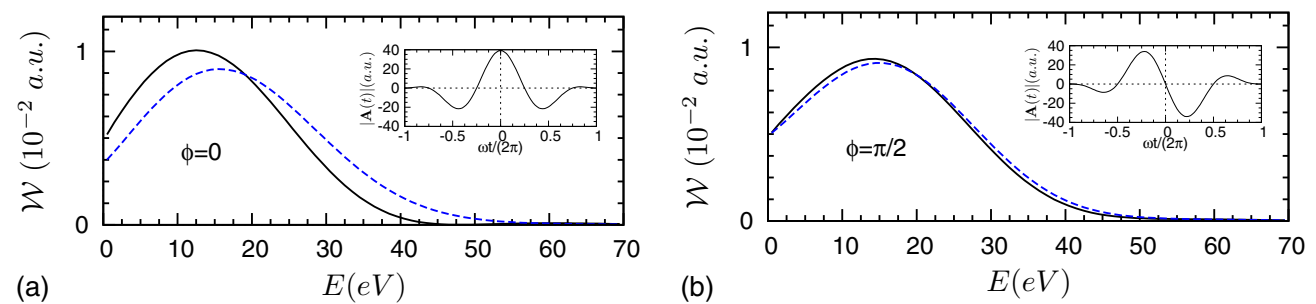

FIG. 3. (Color online) Ionization probability for the $\mathrm{H}$ atom by a linearly polarized pulse with (a) CEP $\phi=0$, (b) CEP $\phi=\pi / 2$, carrier frequency $\omega=36 \mathrm{eV}$, and intensity $I_{0}=5 \times 10^{15} \mathrm{~W} / \mathrm{cm}^{2}$. Each pulse has a duration $\tau=2$ cycles and a cos envelope. Solid and dashed lines: ionization probabilities for electron ejection along $(\alpha=0)$ and opposite to $(\alpha=\pi)$ the direction of linear polarization, respectively. The inset figures show the $z$ axis component of the vector potential $\mathbf{A}(t)$ in each case.

$$
A_{0}(t)=\frac{c \sqrt{I_{0}}}{\omega} \cos ^{2}(\pi t / \tau), \quad-\tau / 2 \leq t \leq \tau / 2
$$

where $c$ is the speed of light. We present results for two carrier frequencies $(\omega=36 \mathrm{eV}$ and $\omega=72 \mathrm{eV})$, two intensities $\left(I_{0}=5 \times 10^{15} \mathrm{~W} / \mathrm{cm}^{2}\right.$ and $\left.I_{0}=2 \times 10^{16} \mathrm{~W} / \mathrm{cm}^{2}\right)$, and a CEP $\phi=0$. The duration $\tau=2 \pi N / \omega$ of each pulse is $N$ $=2$ cycles, which in the case of $\omega=36 \mathrm{eV}$ corresponds to a pulse duration of 230 as. One sees clearly that the results of our analytic PT formulas and those of direct numerical solutions of the TDSE are in excellent agreement. This is not surprising despite the fact that the xuv pulses have quite high intensities. Indeed, the applicability of the PT description of ionization is governed not only by the intensity but also by the smallness of the parameter $\zeta=u_{p} /(\hbar \omega)\left[=I /\left(4 \omega^{3}\right)\right.$ in a.u. $]$, where $u_{p}=e^{2} F^{2} /\left(4 m \omega^{2}\right)$ is the ponderomotive energy [33-35]. (For $\zeta=1$, the divergence of the PT series becomes obvious in view of the closing of the lowest open ionization channel by the ponderomotive potential.) For pulses with frequencies 36 and $72 \mathrm{eV}$ and peak intensities $I_{0}=5$ $\times 10^{15} \mathrm{~W} / \mathrm{cm}^{2}$ and $I_{0}=2 \times 10^{16} \mathrm{~W} / \mathrm{cm}^{2}$, respectively, the parameter $\zeta$ is of the order of $10^{-2}$, thus indicating that we are clearly in the perturbative regime.

\section{B. CEP dependence of angular asymmetries}

In Fig. 3 we illustrate the CEP dependence of the asymmetries in the ionized electron energy distributions for ionization along and opposite to the direction of linear pulse polarization. More specifically, we present the probabilities vs electron energy for ionization of the $\mathrm{H}$ atom by a linearly polarized attosecond pulse with carrier frequency $\omega$
$=36 \mathrm{eV}$, intensity $I_{0}=5 \times 10^{15} \mathrm{~W} / \mathrm{cm}^{2}$, duration $\tau$ $=2$ cycles, and a $\cos ^{2}$ envelope for two different CEPs: $\phi$ $=0$ and $\phi=\pi / 2$. The inset figures show the vector potential [Eq. (71)] of the attosecond pulse in each case. One sees that the asymmetry in the ionized electron angular distribution is maximal for the CEP $\phi=0$ (as is the case also for the corresponding asymmetry in the energy-integrated results, as shown below in Fig. 6). This is in agreement with the TDSE results of Ref. [12]. [Note, however, that in Ref. [12] the vector potential $\mathbf{A}(t)$ for the case of a linearly polarized twocycle pulse is defined in terms of a sine function rather than the cosine function used here [cf. Eq. (71)] so that results in this paper for a CEP $\phi=0$ correspond to results in Ref. [12] for a CEP $\phi=\pi / 2$.] This finding that the asymmetry in the ionized electron angular distribution for the case of linear polarization is maximal for $\phi \approx 0$ and minimal for $\phi \approx \pi / 2$ applies also for other carrier frequencies $\omega$. Hence, in most of the calculations below we used a CEP $\phi=0$. Note that there is an interesting correspondence between the asymmetry of the vector potential of the pulse (i.e., between the maximum magnitudes of its positive and negative values) and the asymmetry in the electron angular distribution: the vector potential is also most asymmetric for a CEP of $\phi=0$.

\section{Sensitivity of CEP-dependent asymmetries to the potential $\boldsymbol{U}(\boldsymbol{r})$}

Comparison of the PT results for the hydrogen atom with those for an electron bound in a ZRP having the same binding energy $\left(E_{n}=-1 / 2\right.$ a.u. $)$ is given in Fig. 4 . In each case the linearly polarized xuv pulse has a $\cos ^{2}$ envelope, a duration of 2 cycles, and a carrier frequency $\omega=36 \mathrm{eV}$. The re-
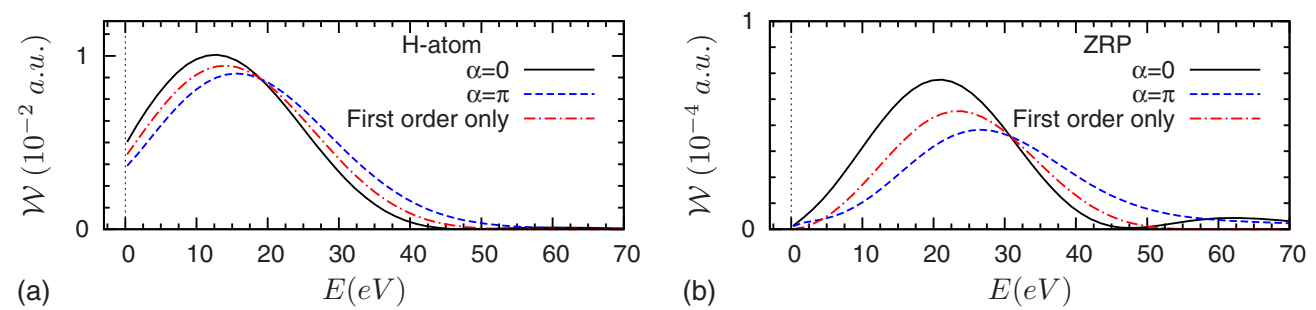

FIG. 4. (Color online) Comparison of ionization probability results for the Coulomb potential (H atom) and the ZRP (with the same binding energy, $E_{n}=-1 / 2$ a.u.) for ionization by a linearly polarized pulse with carrier frequency $\omega=36 \mathrm{eV}, \mathrm{CEP} \phi=0$, intensity $I_{0}=5 \times 10^{15} \mathrm{~W} / \mathrm{cm}^{2}$, duration, $\tau$, of two cycles, and a $\cos ^{2}$ envelope. Results in each panel are given for ionization of electrons both along $(\alpha=0)$ and opposite to $(\alpha=\pi)$ the direction of linear polarization. For purposes of comparison, the first-order PT result, which is spherically symmetric, is also shown. 

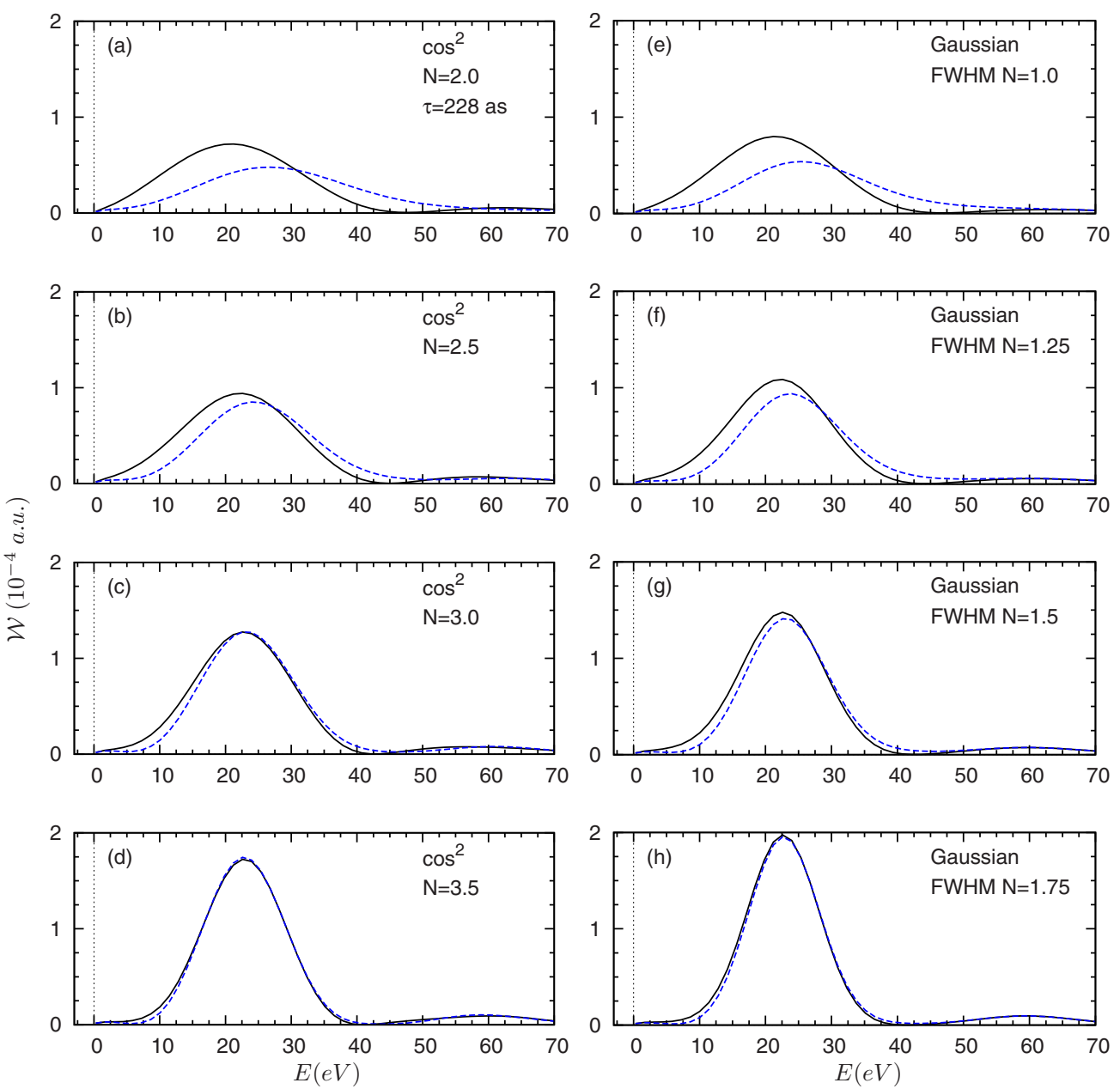

FIG. 5. (Color online) Dependence of the asymmetry of the ionized electron probability distribution (vs energy) on the shape and duration of the xuv pulse envelope for two pulse shapes (cosine squared and Gaussian) and four pulse durations (for each pulse shape). All results are for ionization of an electron bound in a ZRP (with energy $E_{n}=-1 / 2$ ) by a linearly polarized xuv pulse having carrier frequency $\omega=36 \mathrm{eV}$, peak intensity $I_{0}=5 \times 10^{15} \mathrm{~W} / \mathrm{cm}^{2}$, and CEP $\phi=0$. The duration of the Gaussian pulses is the FWHM while that for the $\cos ^{2}$ pulses is the full duration of the pulse. (Note that in each row the two pulses have an equal energy per pulse.) The solid and dashed lines show respectively the ionization probability along (i.e., $\alpha=0$ ) and opposite to (i.e., $\alpha=\pi$ ) the pulse polarization axis.

sults for the ZRP are two orders of magnitude smaller than those for the Coulomb potential owing to the fact that the wave function for the $\mathrm{H}$ atom Coulomb potential is much more localized near the origin compared to that for the ZRP. However, the CEP-dependent asymmetry in the ionized electron angular distribution is much more pronounced in the case of the ZRP, because the second-order term of PT (and hence the first- and second-order interference term) is significantly larger compared to the first-order term. The clear implication of this fact is that for a given pulse intensity negative ions (which can be accurately modeled by a ZRP) are likely to have larger CEP-induced asymmetries than neutral atoms (at least in the region of applicability of PT).

\section{Pulse shape and duration dependence of CEP-dependent asymmetries}

We demonstrate the dependence of the ionized electron asymmetries on the shape and duration of the xuv pulse envelope in Fig. 5, which compares results for xuv pulses hav- ing a $\cos ^{2}$ envelope with those having a Gaussian envelope. In each case the results are for xuv pulse ionization of an electron bound in a ZRP with energy $E_{n}=-1 / 2$ a.u. for linearly polarized pulses having carrier frequency $\omega=36 \mathrm{eV}$, intensity $I_{0}=5 \times 10^{15} \mathrm{~W} / \mathrm{cm}^{2}$, and different pulse durations. The Gaussian envelope for the vector potential of the xuv pulse is defined as

$$
A_{0}(t)=\frac{c \sqrt{I_{0}}}{\omega} \exp \left(-4 \ln 2 \frac{t^{2}}{\tau^{2}}\right) .
$$

Note that while the duration of the cosine squared pulse is defined as the full duration of the pulse, the duration for the Gaussian pulse is defined as its FWHM, which is the reason for the ratio of two between the durations for the two cases. Note, however, that the energy per pulse is the same for the two pulse shapes in each row of the figure, which shows results for four different durations for each pulse shape. The results in Fig. 5 show clearly that the shorter the pulse duration, the larger the asymmetry in the ionized electron distri- 


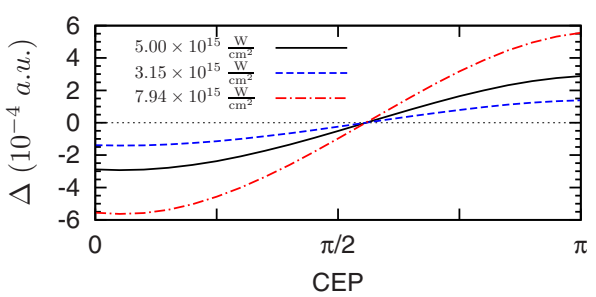

FIG. 6. (Color online) CEP dependence of the energyintegrated asymmetry in the ionized electron distribution for the $\mathrm{H}$ atom along or opposite to the direction of linear polarization of an xuv pulse with carrier frequency $\omega=36 \mathrm{eV}$, two-cycle pulse duration, $\cos ^{2}$ pulse shape, and three different peak intensities: $I_{0}=5 \times 10^{15} \mathrm{~W} / \mathrm{cm}^{2}, \quad I_{0}=3.15 \times 10^{15} \mathrm{~W} / \mathrm{cm}^{2}$, and $I_{0}$ $=7.94 \times 10^{15} \mathrm{~W} / \mathrm{cm}^{2}$.

butions. As discussed in our PT analysis in Secs. II and III above, the reason is that the shorter the pulse duration, the larger the energy region over which there is overlap of the "one-photon" and "two-photon" ionization amplitudes. For pulse durations of 3.5 cycles in the case of a $\cos ^{2}$ envelope and 1.75 cycles FWHM in the case of a Gaussian envelope, the asymmetry almost disappears. One observes also that the ionized electron energy spectra and their asymmetries along the pulse polarization axis are not very sensitive to the pulse shape although a Gaussian envelope gives an ionized electron energy distribution with slightly smaller bandwidth. We conclude that, to a good approximation, either pulse shape can be used to predict results for comparison with experimental data.

\section{E. CEP-induced asymmetry in the energy-integrated angular distribution}

In Fig. 6 we present the CEP dependence of the energyintegrated asymmetry in the electron distribution along or opposite to the direction of linear polarization [cf. Eq. (28)],

$$
\begin{aligned}
\Delta=\int \frac{d \Delta}{d E} d E & =2 \int \operatorname{Re}\left[\left(\gamma_{1}+\gamma_{2}+\gamma_{3}\right) e^{-i \phi}\right] d E \\
& =|K| \cos (\phi+\arg K),
\end{aligned}
$$

where

$$
K \equiv 2 \int\left(\gamma_{1}+\gamma_{2}+\gamma_{3}\right) d E
$$

Of course, $\Delta$ equals also the asymmetry in the ion yield. The main features of the results in Fig. 6 (and their variation for other system parameters) are easily explained from Eq. (74) for $\Delta$ and from the definitions of $\gamma_{i}$ in Eqs. (58)-(60). First, one sees that the CEP dependence of the asymmetry $\Delta$ is sinusoidal, i.e., $\Delta(\phi)=-\Delta(\phi+\pi)$. Second, the amplitude of the oscillation, $K$, depends on intensity as $I^{3 / 2}$. Third, the value of the CEP $\phi$ at which $\Delta=0$ depends on $\arg K$, i.e., on the ratio of the real and imaginary parts of $K$. Fourth, an experimental measurement of the CEP dependence of $\Delta$ can be used to determine the real and imaginary parts of $K$.

\section{F. Angular distributions for elliptically and circularly polarized few-cycle pulses}

In order to characterize the asymmetry in the polarization plane for the cases of elliptical and circular polarization, we define the weighted momentum direction (WMD) of the ionized electron distribution,

$$
\langle\hat{\mathbf{p}}\rangle=\int_{0}^{2 \pi} \hat{\mathbf{p}} \mathcal{W} d \varphi,
$$

where $\varphi$ is the angle in the polarization plane. The meaning of the WMD becomes clearer after substituting in Eq. (76) the ionization probability in Eq. (22) for the general case of elliptical polarization. Defining $\gamma_{ \pm}(\ell)$ as

$$
\gamma_{ \pm}(\ell)=\frac{\pi}{\sqrt{2}}\left[\gamma_{1}+\frac{1}{4}(2 \pm \ell) \gamma_{2} \pm \ell \gamma_{3}\right]
$$

one obtains

$$
\begin{gathered}
\langle\hat{\mathbf{p}}\rangle_{x}=\sqrt{1+\ell} \operatorname{Re}\left[\gamma_{+}(\ell) e^{-i \phi}\right], \\
\langle\hat{\mathbf{p}}\rangle_{y}=\frac{\xi}{\sqrt{1+\ell}} \operatorname{Im}\left[\gamma_{-}(\ell) e^{-i \phi}\right], \\
|\langle\hat{\mathbf{p}}\rangle|=\sqrt{\langle\hat{\mathbf{p}}\rangle_{x}^{2}+\langle\hat{\mathbf{p}}\rangle_{y}^{2}}=\left[(1+\ell) \operatorname{Re}\left[\gamma_{+}^{2}(\ell) e^{-2 i \phi}\right]\right. \\
\left.+(1-\ell) \operatorname{Im}\left[\gamma_{-}^{2}(\ell) e^{-2 i \phi}\right]\right]^{1 / 2}, \\
\tan \theta_{\langle\hat{\mathbf{p}}\rangle}=\frac{\langle\hat{\mathbf{p}}\rangle_{y}}{\langle\hat{\mathbf{p}}\rangle_{x}}=\frac{\xi}{1+\ell} \frac{\operatorname{Im}\left[\gamma_{-}(\ell) e^{-i \phi}\right]}{\operatorname{Re}\left[\gamma_{+}(\ell) e^{-i \phi}\right]} .
\end{gathered}
$$

\section{Numerical results for an elliptically polarized pulse}

In Fig. 7 we demonstrate the polarization dependence of the ionized electron angular distribution in the polarization plane (perpendicular to the direction of light propagation) for a fixed final-state energy, $E=32 \mathrm{eV}$, and a pulse having a two-cycle duration, a $\cos ^{2}$ envelope, a carrier frequency $\omega$ $=36 \mathrm{eV}$, an intensity $I_{0}=5 \times 10^{15} \mathrm{~W} / \mathrm{cm}^{2}$, and a CEP $\phi=0$. The arrows in Fig. 7(a) indicate the WMDs $\langle\hat{\mathbf{p}}\rangle$ for five values of the degree of circular polarization, $\xi$. The fact that the WMDs for values of $\xi$ having opposite signs are different indicates that the probability distribution exhibits a dichroic effect, both for elliptical and for circular polarization. [Note that for $0<|\xi|<1$ both elliptical and circular dichroism effects are present; see Sec. II B.] As may be clearly seen in Fig. 7(b), the magnitude $|\langle\hat{\mathbf{p}}\rangle|$ is maximal for linear polarization $(\xi=0)$ and reaches its minimum for circular polarization $(|\xi|=1)$. Also, one can see that the dependence of the angle $\theta_{\langle\hat{\mathbf{p}}\rangle}$ on the degree of circular polarization, $\xi$, is monotonic, thus providing a means to determine the polarization of the pulse.

\section{Numerical results for a circularly polarized pulse}

For the special case of circular polarization $(|\xi|=1, \ell$ $=0)$, we express the WMD $\langle\hat{\mathbf{p}}\rangle$ in terms of its magnitude, $|\langle\hat{\mathbf{p}}\rangle|=\pi\left|\gamma_{1}+\gamma_{2} / 2\right| / \sqrt{2}$, and its angle in the polarization 

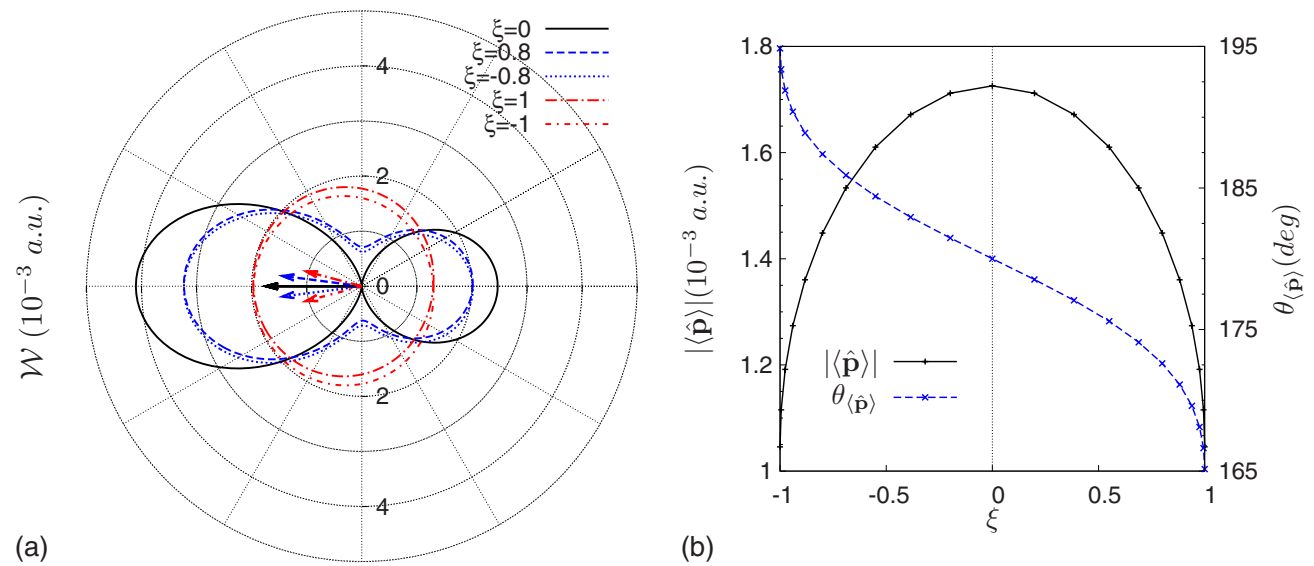

(b)

FIG. 7. (Color online) Polarization dependence of the doubly differential ionization probability of the $\mathrm{H}$ atom in the polarization plane. The ionizing xuv pulse has a two-cycle duration, a $\cos ^{2}$ envelope, a carrier frequency $\omega=36 \mathrm{eV}$, an intensity $I_{0}=5 \times 10^{15} \mathrm{~W} / \mathrm{cm}^{2}, \mathrm{a} \mathrm{CEP}$ $\phi=0$, and a degree of circular polarization $\xi$. All results are for a fixed final-state electron energy, $E=32 \mathrm{eV}$. Figure (a) shows the angular dependence of the doubly differential ionization probability for five values of $\xi$, with the five arrows indicating the weighted momentum direction (WMD) $\langle\hat{\mathbf{p}}\rangle$ [cf. Eq. (76)] for each distribution. Figure (b) shows the magnitude and angle of the WMD $\langle\hat{\mathbf{p}}\rangle$ of the ionized electron distribution as a function of $\xi$.

plane, $\quad \tan \theta_{\langle\hat{\mathbf{p}}\rangle} \equiv \xi \operatorname{Im}\left[\left(\gamma_{1}+\gamma_{2} / 2\right) e^{-i \phi}\right] / \operatorname{Re}\left[\left(\gamma_{1}+\gamma_{2} / 2\right) e^{-i \phi}\right]$ $=\xi \tan \left[\phi+\arg \left(\gamma_{1}+\gamma_{2} / 2\right)\right]$. As discussed above, the CEP for circular polarization may be set to zero, $\phi=0$, by rotation of the laboratory system of coordinates. In this case the angle $\theta_{\langle\hat{\mathbf{p}}\rangle}$ characterizes the ratio of the imaginary and real parts of $\gamma_{1}+\gamma_{2} / 2$.

In Fig. 8 we show results for circularly polarized twocycle pulses, each having a $\cos ^{2}$ envelope, carrier frequency $\omega=36 \mathrm{eV}$, intensity $I_{0}=5 \times 10^{15} \mathrm{~W} / \mathrm{cm}^{2}$, and CEP $\phi=0$. The first and second rows of Fig. 8 correspond, respectively, to right and left circular polarizations. The first column shows the ionized electron distribution in the polarization plane for several different final-state energies. The asymmetry in the ionized electron angular distribution manifests itself as a shift of the distribution away from the center of coordinates as well as a deformation from a perfect circle (which would be the case in the absence of the interference term). Values of the WMDs for each of the final-state energies are shown as the arrows in Figs. 8(a) and 8(c).

The energy dependences of the magnitude $|\langle\hat{\mathbf{p}}\rangle|$ and the angle $\theta_{\langle\hat{\mathbf{p}}\rangle}$ of the WMD defined in Eqs. (80) and (81) are given for the cases of right and left circular polarization in Figs. 8(b) and 8(d). The ionized electron distribution for right circular polarization is symmetric under reflection [with respect to the (horizontal) $x$ axis] with the distribution for left circular polarization, as may be seen from Eq. (33). Indeed, changing the sign of circular polarization is equivalent to changing the sign of the angle $\varphi$, i.e., $\varphi \rightarrow-\varphi$. The magnitude $|\langle\hat{\mathbf{p}}\rangle|$ has a minimum around $E=20 \mathrm{eV}$, which is why the arrow representing the WMD for this energy is not visible in Figs. 8(a) and 8(c). Also, for energies $E>20 \mathrm{eV}$, the angle $\theta_{\langle\hat{\mathbf{p}}\rangle}$ is nearly constant, implying that the ratio of the imaginary and real parts of $\gamma_{1}+\gamma_{2} / 2$ is nearly constant.

The results in Figs. 8(a) and 8(c) show also that the maximum of the ionization probability for a given final-state energy is shifted from the direction at which vector potential, $\mathbf{A}(t)$, reaches its maximum. The shift angle equals $\theta_{C}-\phi[\mathrm{cf}$. Eqs. (33) and (34)], where $\theta_{C}$ depends on the energy of the final state. The values of $\theta_{C}$ are given in Figs. 8(b) and 8(d) since for a CEP $\phi=0$ the WMD angle $\theta_{\langle\hat{\mathbf{p}}\rangle}=\theta_{C}$. This angular shift originates from the dichroic part of the interference term in the ionization probability, i.e., the term $\sim \xi$ in Eq. (22). It is the analog of the one found numerically in Ref. [36] by solving the TDSE for ATI by a few-cycle circularly polarized $800 \mathrm{~nm}$ laser pulse.

\section{G. Dependence of the asymmetry $d \Delta / d E$ on electron energy}

For a linearly polarized pulse, $\langle\hat{\mathbf{p}}\rangle_{y}=0$ and $\langle\hat{\mathbf{p}}\rangle_{x}$ in Eq. (78) is proportional to the difference of the ionization probabilities along $(\alpha=0)$ and opposite to $(\alpha=\pi)$ the polarization axis [cf. Eqs. (28) and (29) and Fig. 1]:

$$
\frac{2}{\pi}\langle\hat{\mathbf{p}}\rangle_{x}=\frac{d \Delta}{d E}=2\left|\gamma_{1}+\gamma_{2}+\gamma_{3}\right| \cos \left(\phi+\theta_{L}\right) .
$$

This asymmetry in the electron distribution along the direction of linear polarization is in fact exactly twice the interference term in Eq. (38) evaluated at $\alpha=0$. In Fig. 9 we present this asymmetry as a function of the ionized electron's kinetic energy, $E$. The asymmetry $d \Delta / d E$ reaches its maximum and minimum values at energies $E \approx 5.5 \mathrm{eV}$ and $E$ $\approx 32.5 \mathrm{eV}$, respectively; it equals zero at $E \approx 19 \mathrm{eV}$. For these three energies, we present in Table I the real and imaginary parts of the first- and second-order matrix elements, $\alpha_{ \pm}$ and $M_{i}^{ \pm \pm}$[cf. Eqs. (45)-(51)], as well as the first- and second-order amplitudes, $A_{1}$ and $A_{2}$, for ionization along the direction of linear polarization (i.e., $\alpha=0$ ) of an xuv attosecond pulse with carrier frequency $\omega=36 \mathrm{eV}$, peak intensity $I_{0}=5 \times 10^{15} \mathrm{~W} / \mathrm{cm}^{2}$, two-cycle pulse duration, and a $\cos ^{2}$ pulse shape.

Table I shows that those matrix elements corresponding to emission of one photon in the first-order amplitude $A_{1}$ (i.e., $\left.\alpha_{-}\right)$and to emission of two photons in the second-order amplitude $A_{2}$ (i.e., $M_{i}^{--}$) are small compared to the other matrix elements so that they can be neglected. Specifically, $\alpha_{-}$contributes only about $0.4 \%$ to the real and imaginary parts of $A_{1}$ 

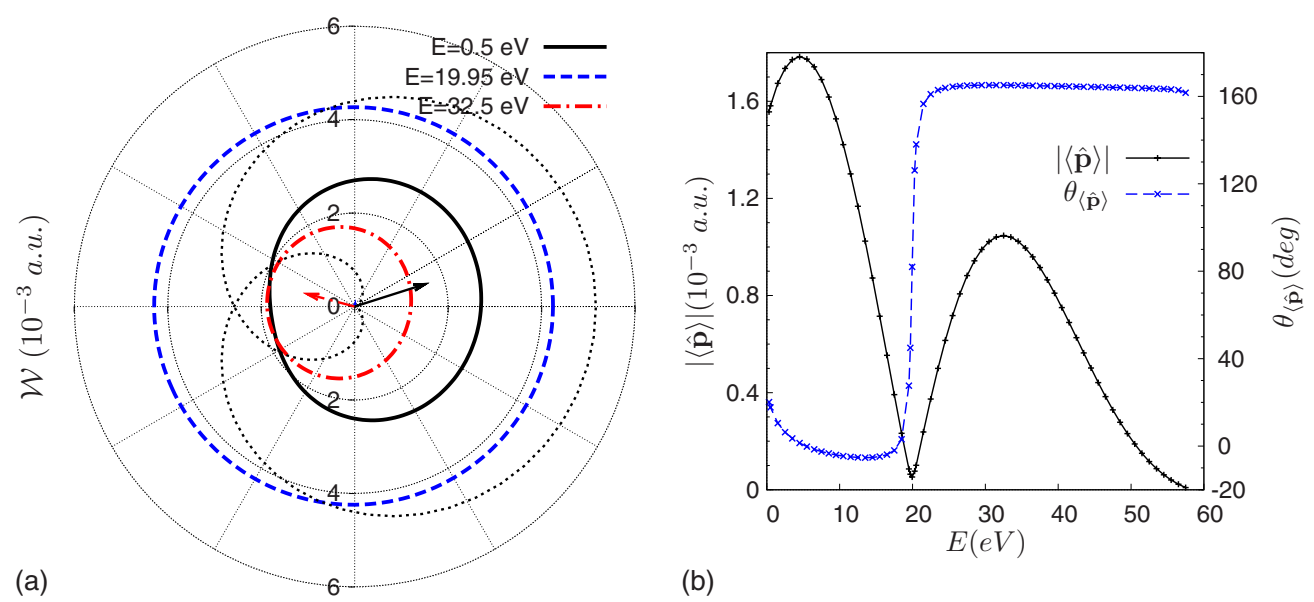

(b)
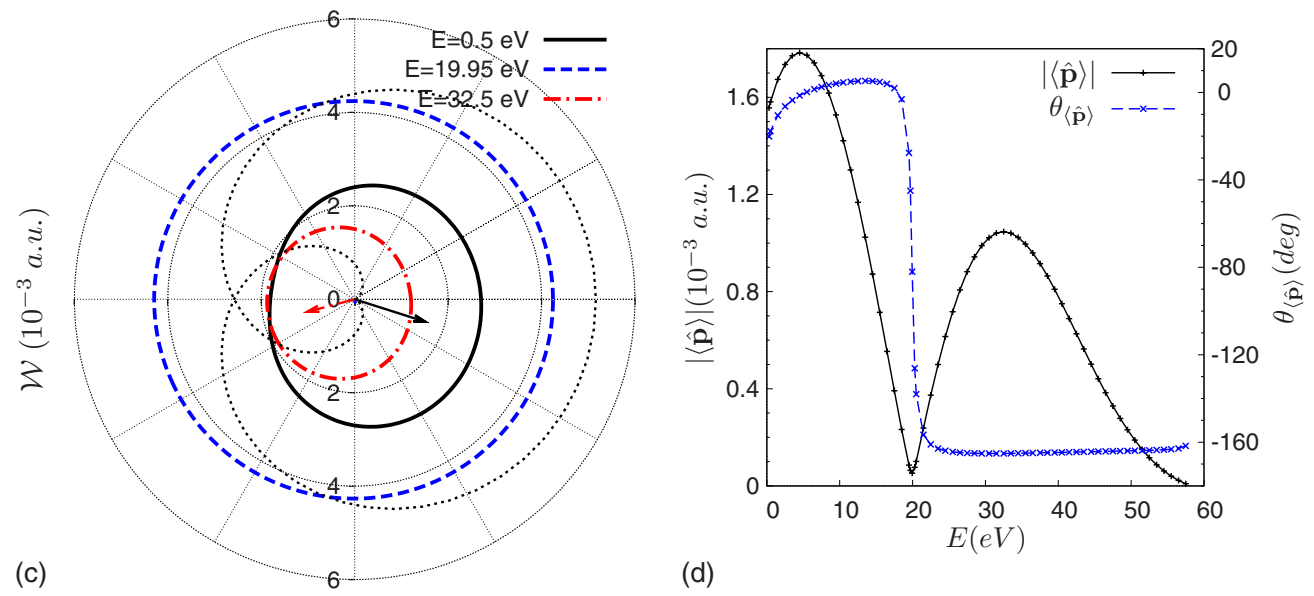

(d)

FIG. 8. (Color online) Ionization probability of the $\mathrm{H}$ atom by right and left circularly polarized, two-cycle xuv pulses having cos ${ }^{2}$ envelopes, carrier frequency $\omega=36 \mathrm{eV}$, intensity $I_{0}=5 \times 10^{15} \mathrm{~W} / \mathrm{cm}^{2}$, and CEP $\phi=0$. Panels (a) and (c) show the electron angular distribution for right and left circular polarization, respectively, for each of three final-state energies as a function of ejection angle in the polarization plane. The arrows in these figures indicate the weighted momentum direction (WMD) $\langle\hat{\mathbf{p}}\rangle$ [cf. Eq. (76)] for each distribution. Panels (b) and (d) show the energy dependences of the magnitude, $|\langle\hat{\mathbf{p}}\rangle|$, and the phase, $\arg (\langle\hat{\mathbf{p}}\rangle) \equiv \theta_{\langle\hat{\mathbf{p}}\rangle}$, of the WMD $\langle\hat{\mathbf{p}}\rangle$ for right and left circular polarization, respectively. The black dotted lines in panels (a) and (c) show the vector potential in atomic units, $\frac{1}{c} \mathbf{A}(t)$.

at all three energies, while $M_{0+2}^{--}-3 M_{2}^{--}$contributes less than $0.3 \%$ to the real and imaginary parts of $A_{2}$, except at the energy $E=19 \mathrm{eV}$ (at which $d \Delta / d E \approx 0$ ) where its contribution is $1.8 \%$ of a very small value of $\operatorname{Re} A_{2}$. (For a more detailed description of the role of the emission terms, see Appendix C.)

The contribution of single-photon emission (accompanied by single-photon absorption) to the second-order amplitude

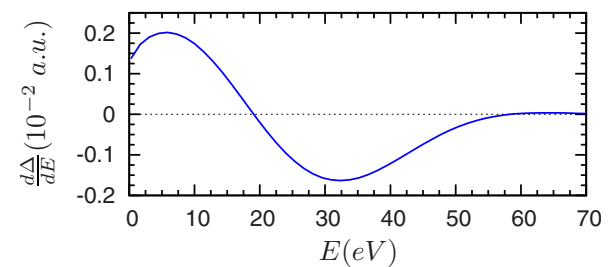

FIG. 9. (Color online) Asymmetry $d \Delta / d E$ [cf. Eqs. (28) and (82)] in the distribution of electrons ionized with kinetic energy $E$ from the $\mathrm{H}$ atom along or opposite to the direction of linear polarization of an xuv pulse with carrier frequency $\omega=36 \mathrm{eV}$, peak intensity $I_{0}=5 \times 10^{15} \mathrm{~W} / \mathrm{cm}^{2}$, two-cycle pulse duration, $\cos ^{2}$ pulse shape, and CEP $\phi=0$.
$A_{2}$, however, is significant, as shown by the matrix elements in Table I and by the amplitudes, ionization probabilities, and asymmetries shown in Fig. 10. Specifically, in the region of small electron energies at which the asymmetry takes its maximum positive value $(E=5.5 \mathrm{eV})$ [cf. Fig. $10(\mathrm{c})]$, the largest contributions to the second-order amplitude $A_{2}$ come from the terms involving emission-absorption plus absorption-emission of two photons: $M_{0+2}^{+-}-3 M_{2}^{+-}$[cf. Fig. 10 (a)]. In contrast, in the region of high electron energies at which the asymmetry takes its maximum negative value $(E=32.5 \mathrm{eV})$, the dominant contribution to $A_{2}$ comes from terms involving absorption of two photons, $M_{0+2}^{++}-3 M_{2}^{++}$. This difference in the most important contributions to $A_{2}$ at low and high energies may be understood quite simply: at higher energies, the electron energy $E$ is closer to the twophoton resonance, $E_{n}+2 \omega$, at which the two-photon absorption amplitude is largest; at the lower energy, the electron energy $E$ is closer to the initial state energy, $E_{n}+\omega-\omega$, at which the absorption-emission terms are largest. The large bandwidth of the few-cycle pulse allows interference between $A_{1}$ and these two very different second-order contributions to $A_{2}$. 
TABLE I. Real and imaginary parts of the first- and second-order transition amplitudes $A_{1}$ and $A_{2}$ [cf. Eqs. (38), (45), and (46)] and their respective component matrix elements $\mathcal{A}^{ \pm}$[cf. Eq. (47)] and $M_{i}^{ \pm \pm}[\mathrm{cf}$. Eqs. (48)-(51)] for ionization of atomic $\mathrm{H}$ with ejection of electrons at the angle $\alpha=0$ along the direction of linear polarization of an xuv pulse with carrier frequency $\omega=36 \mathrm{eV}$, peak intensity $I_{0}=5 \times 10^{15} \mathrm{~W} / \mathrm{cm}^{2}$, two-cycle pulse duration, and a $\cos ^{2}$ pulse shape.

\begin{tabular}{|c|c|c|c|c|c|c|}
\hline \multirow[b]{2}{*}{$E$} & \multicolumn{2}{|c|}{$5.5 \mathrm{eV}$} & \multicolumn{2}{|c|}{$19 \mathrm{eV}$} & \multicolumn{2}{|c|}{$32.5 \mathrm{eV}$} \\
\hline & $10^{4} \times \operatorname{Re}$ & $10^{4} \times \operatorname{Im}$ & $10^{4} \times \mathrm{Re}$ & $10^{4} \times \operatorname{Im}$ & $10^{4} \times \mathrm{Re}$ & $10^{4} \times \operatorname{Im}$ \\
\hline$\alpha_{+}$ & -678.962 & 813.847 & -788.087 & 329.215 & -426.310 & 127.685 \\
\hline$\alpha_{-}$ & -2.961 & 3.549 & -2.780 & 1.161 & 1.814 & -0.543 \\
\hline$A_{1}$ & -681.923 & 817.396 & -790.867 & 330.376 & -424.496 & 127.142 \\
\hline$M_{0+2}^{++}$ & 0.959 & 3.970 & -7.025 & 2.249 & -10.975 & -2.752 \\
\hline$M_{0+2}^{+-}$ & -36.254 & 16.828 & -7.036 & 1.173 & -0.515 & -0.030 \\
\hline$M_{0+2}^{--}$ & -0.006 & 0.075 & 0.012 & -0.006 & -0.030 & -0.003 \\
\hline$-3 M_{2}^{++}$ & 7.298 & -20.848 & 53.546 & -18.037 & 88.565 & -7.560 \\
\hline$-3 M_{2}^{+-}$ & -15.734 & 60.665 & -36.740 & 21.959 & -16.150 & 5.813 \\
\hline$-3 M_{2}^{--}$ & -0.004 & -0.103 & -0.036 & 0.007 & 0.011 & -0.001 \\
\hline$A_{2}$ & -43.741 & 60.587 & 2.722 & 7.344 & 60.933 & -4.530 \\
\hline$\theta_{L}(\operatorname{deg})$ & \multicolumn{2}{|c|}{-4.0} & \multicolumn{2}{|c|}{-87.7} & \multicolumn{2}{|c|}{-167.6} \\
\hline$\left|A_{1}^{*} A_{2}\right| \times 10^{4}$ & \multicolumn{2}{|c|}{7.955} & \multicolumn{2}{|c|}{0.671} & \multicolumn{2}{|c|}{2.708} \\
\hline
\end{tabular}

At $E \approx 19 \mathrm{eV}$ the interference term (from which the asymmetry originates) is zero [cf. Figs. 10(b) and 10(c)], i.e., $\operatorname{Re} A_{1}^{*} A_{2}=\operatorname{Re} A_{1} \operatorname{Re} A_{2}+\operatorname{Im} A_{1} \operatorname{Im} A_{2}=0$. Note that although the amplitude $A_{2}$ is almost an order of magnitude smaller at this energy than at energies at which the interference term reaches its maximum magnitudes, the real and imaginary parts of $A_{2}$ are usually never zero simultaneously, i.e., $\left|A_{2}\right|$ $\neq 0$. From Table I one sees that the change in sign of the asymmetry (or interference term) around $E \approx 19 \mathrm{eV}$ is due to a change in sign of the real and imaginary parts of the second-order amplitude $A_{2}$ with increasing energy. The amplitude $A_{1}$ does not change sign over this range of energies.
Finally, as shown in Fig. $10(\mathrm{a})$, near $E \approx 19 \mathrm{eV}$, the magnitudes of the absorption-and-emission and the two-photon absorption contributions to $\left|A_{2}\right|$ are equal in magnitude.

As demonstrated by the results for the doubly differential ionization probabilities in Fig. 3 and by the energy-integrated asymmetry [cf. Eqs. (74) and (75)] in Fig. 6, the asymmetry in the ionized electron distribution along the linear polarization axis of the pulse takes its largest magnitude at the CEP $\phi=0$. This fact may be explained using Eq. (82) and the data in Table I for $A_{1}, A_{2}$, and $\theta_{L}$ (for the angle $\alpha=0$ ) at the three values of $E$ given there. Note first that $\left.p A_{1}^{*} A_{2}\right|_{\alpha=0}=\gamma_{1}+\gamma_{2}$ $+\gamma_{3}$ and that the sign of the asymmetry is given by that of

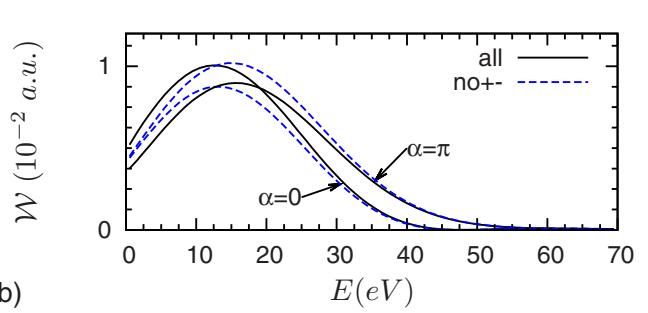

(a)
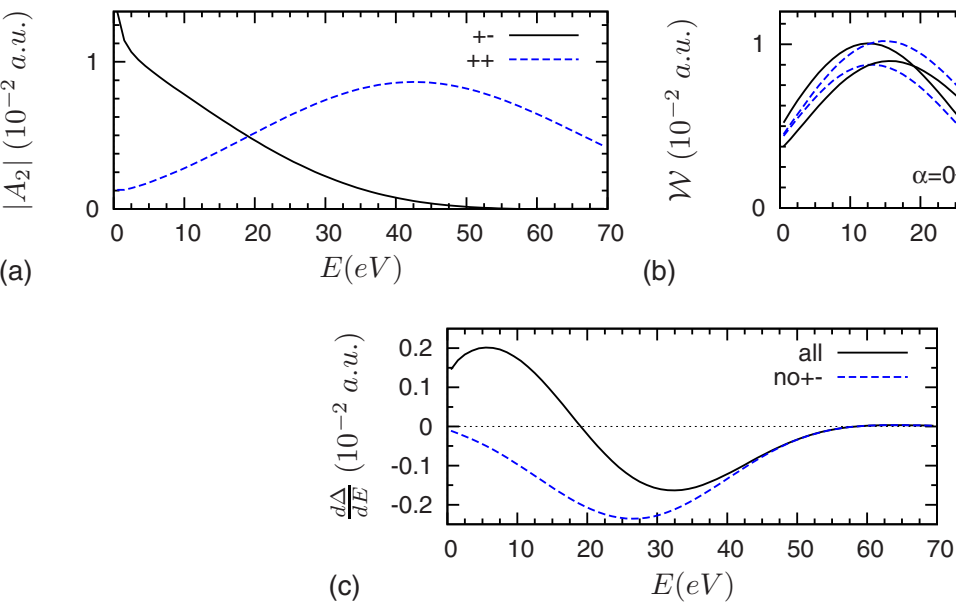

FIG. 10. (Color online) Contribution of terms involving emission-absorption plus absorption-emission of two photons [i.e., $M_{i}^{+-}$terms in Eq. (46)] to the second-order amplitude $A_{2}$ for ionization of the $\mathrm{H}$ atom by a few-cycle xuv pulse having the same parameters as in Fig. 9 . (a) The two most important contributions to the second-order amplitude for electron ejection along $\alpha=0$ : the solid (black) line is $\left|A_{2}\right|$ with only $M_{i}^{+-}$terms (for absorption and emission) included; the dashed (blue) line is $\left|A_{2}\right|$ with only $M_{i}^{++}$terms (for two-photon absorption) included. (b) The ionization probability, $\mathcal{W}$, without $M_{i}^{+-}$terms in $A_{2}$ is compared to the result with all terms included in $A_{2}$. (c) The asymmetry [see Eq. (28)] corresponding to each of the two ionization probabilities shown in panel (b). 
$\cos \left(\phi+\theta_{L}\right)$. The numerical values of $\theta_{L}$ and $\left|A_{1}^{*} A_{2}\right|$ for three electron kinetic energies $E$ are given in the last two rows of Table I. For $E=5.5 \mathrm{eV}$ and $\theta_{L}=-4.0^{\circ}$ so that $\cos \left(\phi+\theta_{L}\right)$ $\approx 1$ for a CEP $\phi=0$ and $\cos \left(\phi+\theta_{L}\right) \approx 0$ for a CEP $\phi=\pi / 2$. For $E=19 \mathrm{eV}$ and $\theta_{L}=-87.7^{\circ}$ so that $\cos \left(\phi+\theta_{L}\right) \approx 0$ for a CEP $\phi=0$ and $\cos \left(\phi+\theta_{L}\right) \approx 1$ for a CEP $\phi=\pi / 2$; however, $\left|A_{1}^{*} A_{2}\right|$ is very small at this energy so the asymmetry is small. For $E=32.5 \mathrm{eV}$ and $\theta_{L}=-167.7^{\circ}$ so that $\cos \left(\phi+\theta_{L}\right) \approx-1$ for a CEP $\phi=0$ and $\cos \left(\phi+\theta_{L}\right) \approx 0$ for a CEP $\phi=\pi / 2$. Thus, the magnitude of the asymmetry is maximal around $\phi=0$ and minimal around $\phi=\pi / 2$, which is why many of the results we have presented are for pulses having $\phi=0$.

\section{SUMMARY AND CONCLUSIONS}

In summary, in this paper we have analyzed the ionization of an atomic system by a few-cycle attosecond pulse using a PT approach. Our main analytical results include: (i) a general parametrization of the ionization probability (using rotational invariance and symmetry arguments) in terms of the physical vectors of the problem, i.e., the polarization vector $\mathbf{e}$ and the direction of the ionized electron momentum $\hat{\mathbf{p}}$, as well as the CEP of the pulse [cf. Eqs. (16) and (17)]; (ii) identification of circular and elliptic dichroism terms in the general expression for the ionization probability [cf. Eqs. (23)-(26)]; (iii) in the general case of elliptic polarization [cf. Eq. (20)] as well as in the special cases of linear [cf. Eq. (29)] and circular [cf. Eq. (33)] polarizations, the asymmetries in the ionized electron angular distributions are shown to depend not only on the CEP of the pulse, but also on the phase of certain dynamical parameters; (iv) in Sec. III we express the dynamical parameters in terms of radial matrix elements (within a single-active-electron model of the atom) for the cases of initial $s$ and $p$ states, and in Appendixes A and $\mathrm{B}$ we give analytic expressions for these radial matrix elements for both a ZRP and a Coulomb potential, respectively.

In Sec. IV we have presented numerous numerical results based on our analytically derived formulas in Secs. II and III. Our main ones include: (i) comparison of results of our PT analysis with results of numerical solution of the timedependent Schrödinger equation in Ref. [12] show excellent quantitative agreement, indicating the validity of a PT approach (for the pulse intensities considered); (ii) results for two different pulse shapes (cosine-squared and Gaussian) show good qualitative agreement, indicating that either may be employed in comparisons with experimental results; (iii) for the case of an arbitrary pulse polarization, Fig. 7(b) shows that the weighted momentum direction [cf. Eq. (76)] in the polarization plane is monotonically related to the xuv pulse polarization, thus providing a means to determine this polarization; (iv) for the case of circular polarization, Fig. 8 shows that the asymmetry in the angular distribution in the polarization plane is governed not only by the CEP but also by the phase of the relevant dynamical parameters; (v) in Fig. 10 we have shown that the asymmetry in the electron angular distributions originates from different contributions to the second-order transition amplitude at low and high electron energies, i.e., at low energies the emission- absorption contributions dominate, whereas at high energies the two-photon absorption contribution dominates; moreover, in Appendix $\mathrm{C}$ we evaluate the magnitudes of all other terms involving photon emission, showing that they make only very small contributions to the ionization probability.

We emphasize that although our numerical results in this paper were presented for a fixed carrier frequency $\omega$ of the pulse $\mathbf{F}(t)$ in Eq. (5), all our ab initio parametrizations in Sec. II, as well as explicit quantum expressions for the dynamical parameters in Sec. III, are valid also for chirped pulses, i.e., those with time-dependent carrier frequency, $\omega$ $=\omega(t)$ [since the carrier frequency enters only into the Fourier components $\hat{F}^{ \pm}(\varepsilon)$ in Eq. (8)]. Significant modifications of the asymmetries in ionized electron angular distributions for the case of chirped pulses has been demonstrated recently based on the numerical solution of the TDSE for ionization of the $\mathrm{H}$ atom by a linearly polarized chirped xuv pulse [39]. Application of our PT formulas to the analysis of chirpedpulse attosecond photoionization will be published elsewhere.

In conclusion, in this paper we have analyzed a number of physical phenomena in a new regime: nonlinear attosecond science. Specifically, we have analyzed those phenomena applicable to ionization of an atom by a few-cycle attosecond pulse that originate from interference of first- and secondorder transition amplitudes. As is well known, this interference, which depends on transition amplitude phases, provides the possibility for quantum control of electron motion. Experimental study of these phenomena, however, will require achieving xuv pulses of somewhat higher intensity than is currently possible. Given the rapid progress in experimental attosecond science, we are hopeful that achieving such intensities is only a matter of time.

\section{ACKNOWLEDGMENTS}

This work was supported in part by the U.S. Department of Energy, Office of Science, Division of Chemical Sciences, Geosciences, and Biosciences, under Grant No. DE-FG0396ER14646, by the U.S. National Science Foundation under Grant No. PHY05-51164, and by the Russian Foundation for Basic Research under Grants No. 07-02-00574 and No. 1002-00235. A.F.S. acknowledges the hospitality of the Kavli Institute for Theoretical Physics at the University of California at Santa Barbara, where part of this paper was prepared.

\section{APPENDIX A: ANALYTICAL RESULTS FOR THE ZRP}

For the case of the ZRP, which supports a single bound $s$ state with energy $E_{0}=-\kappa^{2} / 2, \psi_{\kappa}(\mathbf{r})=\sqrt{\kappa /(2 \pi)} r^{-1} \exp (-\kappa r)$, the first- and second-order transition amplitudes can be calculated analytically. For the second-order amplitude $A_{2}$, we performed these calculations using Eq. (42) in terms of the non-stationary Green's function $\mathcal{G}\left(\mathbf{r}, \mathbf{r}^{\prime} ; t-t^{\prime}\right)$ for a ZRP without use of the Fourier expansion for $\mathbf{F}(t)$. The resulting parametrizations for $A_{1}$ and $A_{2}$ are as follows:

$$
A_{1}=-\frac{i \sqrt{\kappa}}{2 \pi \mathcal{E}^{2}}[\mathbf{p} \cdot \hat{\mathbf{F}}(\mathcal{E})],
$$




$$
\begin{aligned}
A_{2}= & a_{0} \mathcal{D}^{(0)}(\mathcal{E})+a_{1} \sum_{i, j} \hat{p}_{i} \hat{p}_{j} \mathcal{D}_{i, j}^{(0)}(\mathcal{E})+b_{0} \mathcal{D}^{(1)}(\mathcal{E})+b_{1} \sum_{i, j} \hat{p}_{i} \hat{p}_{j} \mathcal{D}_{i, j}^{(1)} \\
& \times(\mathcal{E})+c_{0} C(\mathcal{E}),
\end{aligned}
$$

where $\mathcal{E}=E-E_{0}=\left(p^{2}+\kappa^{2}\right) / 2$,

$$
\begin{gathered}
a_{0}=-\frac{2 \sqrt{\kappa}}{3 \pi \mathcal{E}^{2}}\left(1-\frac{i \kappa p}{(\kappa+i p)^{2}}\right), \quad a_{1}=\frac{\sqrt{\kappa} p^{2}}{\pi \mathcal{E}^{3}}, \\
b_{0}=\frac{\sqrt{\kappa}}{6 \pi \mathcal{E}^{2}}\left(\frac{\kappa^{4}+p^{4}}{2 \mathcal{E}}+i \kappa p\right), \quad b_{1}=-\frac{\sqrt{\kappa} p^{2}}{2 \pi \mathcal{E}^{2}}, \\
c_{0}=\frac{i \sqrt{2 \kappa}}{3 \pi(\kappa+i p)}, \\
\mathcal{D}_{i, j}^{(0)}(\mathcal{E})=\frac{i}{2 \pi} \int_{-\infty}^{\infty} d t \int_{0}^{\infty} d \tau F_{i}(t) F_{j}(t-\tau) e^{i \mathcal{E}(t-\tau)}, \\
\mathcal{D}_{i, j}^{(1)}(\mathcal{E})=\frac{1}{2 \pi} \int_{-\infty}^{\infty} d t \int_{0}^{\infty} d \tau F_{i}(t) F_{j}(t-\tau) e^{i \mathcal{E}(t-\tau)} \tau, \\
\mathcal{D}^{(0)}(\mathcal{E})=\sum_{i} \mathcal{D}_{i, i}^{(0)}(\mathcal{E}), \quad \mathcal{D}^{(1)}(\mathcal{E})=\sum_{i} \mathcal{D}_{i, i}^{(1)}(\mathcal{E}), \\
C(\mathcal{E})=\int_{\left|E_{0}\right|}^{\infty} \frac{\hat{\mathbf{F}}\left(\mathcal{E}-\mathcal{E}^{\prime}\right) \cdot \hat{\mathbf{F}}\left(\mathcal{E}^{\prime}\right)}{\left(\mathcal{E}^{\prime}-\mathcal{E}-i 0\right)^{2} \mathcal{E}^{\prime 2}}\left(\mathcal{E}^{\prime}-\left|E_{0}\right|\right)^{3 / 2} d \mathcal{E}^{\prime} .
\end{gathered}
$$

The connection of these ZRP results to the general expressions (45)-(53) for $A_{1}$ and $A_{2}$ involving the radial matrix elements is as follows:

$$
\begin{gathered}
\mathcal{A}_{p 1}=-\frac{i \sqrt{\kappa}}{2 \pi \mathcal{E}^{2}}, \\
\int d \varepsilon\left[\hat{\mathbf{F}}(E-\varepsilon) \cdot \hat{\mathbf{F}}\left(\varepsilon-E_{n}\right)\right]\left[\mathcal{M}_{p 0}(\varepsilon)+\mathcal{M}_{p 2}(\varepsilon)\right] \\
=a_{0} \mathcal{D}^{(0)}+b_{0} \mathcal{D}^{(1)}+c_{0} C(\mathcal{E}), \\
-3 \int d \varepsilon[\hat{\mathbf{p}} \cdot \hat{\mathbf{F}}(E-\varepsilon)]\left[\hat{\mathbf{p}} \cdot \hat{\mathbf{F}}\left(\varepsilon-E_{n}\right)\right] \mathcal{M}_{p 2}(\varepsilon) \\
=a_{1} \sum_{i, j} \hat{p}_{i} \hat{p}_{j} \mathcal{D}_{i, j}^{(0)}+b_{1} \sum_{i, j} \hat{p}_{i} \hat{p}_{j} \mathcal{D}_{i, j}^{(1)},
\end{gathered}
$$

where the integrals over the intermediate state energy $\varepsilon$ were evaluated analytically. We verified the validity of Eqs. (A11) and (A12) using known analytic results for the second-order radial matrix elements in $\mathcal{M}_{p l}(\varepsilon)$ [cf. Eq. (53)] for the case of a ZRP [26].

As is seen from Eqs. (A1)-(A9), for the case of a ZRP the dependence of the amplitudes $A_{1}$ and $A_{2}$ on the atomic and laser parameters factorizes, with the exception of the scalar parameter $C(\mathcal{E})$ in Eq. (A9), which cannot be so factorized and depends on the binding energy $\left|E_{0}\right|$. In particular, the $\mathcal{D}$ functions characterize the second-order characteristics of the pulse; specifically, they are a convolution or cross correlation of the pulse with a weighting factor. (The first-order characteristics are represented by the Fourier transform of the pulse.) For the case of a monochromatic field, i.e., $\mathbf{F}(t)$ $=F_{0} \operatorname{Re}\left[\mathbf{e} e^{-i \omega t}\right]$, the $\mathcal{D}$ functions become proportional to delta functions:

$$
\begin{gathered}
\mathcal{D}_{i, j}^{(0)}(\mathcal{E})=\frac{e_{i} e_{j}}{\omega} \delta\left(E-E_{0}-2 \omega\right), \\
\mathcal{D}_{i, j}^{(1)}(\mathcal{E})=-\frac{e_{i} e_{j}}{\omega^{2}} \delta\left(E-E_{0}-2 \omega\right) .
\end{gathered}
$$

These delta functions enforce energy conservation for absorption of two photons. Since $\hat{\mathbf{F}}(\mathcal{E})$ in Eq. (A1) is proportional to $\delta\left(E-E_{0}-\omega\right)$ for a monochromatic field, it is obvious that the interference of the amplitudes $A_{1}$ and $A_{2}$ decreases rapidly with decreasing pulse bandwidth and vanishes for a monochromatic pulse.

\section{APPENDIX B: ANALYTICAL RESULTS FOR THE COULOMB POTENTIAL}

For the case of ionization of the $1 s$ state of a Coulomb potential with nuclear charge $Z$ to a continuum state with energy $E=p^{2} / 2$ and angular momentum $l=1$, the first-order radial matrix element [Eq. (52)] has the following analytic form:

$$
\mathcal{A}_{p 1}=-\frac{4 i}{\pi p^{3}} \frac{C_{p 1} a^{5 / 2}}{\left(a^{2}+1\right)^{3}}\left(\frac{i a-1}{i a+1}\right)^{i a},
$$

where

$$
a=Z / p, \quad C_{p l}=\sqrt{2 p} e^{\pi a / 2} \Gamma(l+1-i a) .
$$

The second-order matrix elements in Eq. (53) for the case of ionization of the $1 s$ state of the Coulomb potential are a combination of hypergeometric functions of two variables [the Appell functions $F_{1}\left(a ; b, c ; d ; x, x^{\prime}\right)$ [37]], as shown, e.g., by Eq. (51) in Ref. [38], which is applicable for twophoton ionization of the $1 s$ state. (Note that in Ref. [38] the matrix elements are given in the velocity gauge and are related to the matrix elements in the length gauge through the relation given after Eq. (35) in Ref. [38].) Using these relations, the second-order radial matrix elements in Eq. (53) for the $1 s$ state of the Coulomb potential are given by

$$
\begin{aligned}
\mathcal{M}_{p 0}(\varepsilon)= & -\frac{8 i}{3 \pi^{2} p^{3 / 2}} \frac{C_{p 0} a^{5 / 2}}{(E-\varepsilon)\left(\varepsilon+Z^{2} / 2\right)} \\
& \times[(1-i a)(2-i a) F(0)-(1+i a)(2+i a) F(2)],
\end{aligned}
$$

$$
\mathcal{M}_{p 2}(\varepsilon)=\frac{8 \sqrt{2} i}{3 \pi^{2} p^{3 / 2}} \frac{C_{p 2} 2^{5 / 2}}{(E-\varepsilon)\left(\varepsilon+Z^{2} / 2\right)}[F(0)-F(2)]
$$

where $\varepsilon$ is the intermediate state energy, $E=p^{2} / 2$, and 


$$
\begin{gathered}
F(m)=\frac{\nu^{5}}{(2-Z \nu)(1+Z \nu)^{4}} \frac{(\nu-i / p)^{i a-3+m}}{(\nu+i / p)^{i a+1+m}} \\
\times F_{1}\left(2-Z \nu ;-i a+3-m, i a+1+m ; 3-Z \nu ; x, x^{\prime}\right), \\
\nu=\frac{1}{\sqrt{-2 \varepsilon-i 0}}, \\
x=\frac{Z \nu-1}{Z \nu+1} \frac{\nu+i / p}{\nu-i / p}, \quad x^{\prime}=\frac{Z \nu-1}{Z \nu+1} \frac{\nu-i / p}{\nu+i / p}
\end{gathered}
$$

In a similar way, using the results in Ref. [38], second-order radial matrix elements $\left\langle p l\left|r g_{L}\left(E ; r, r^{\prime}\right) r^{\prime}\right| n 1\right\rangle$ in Eq. (70) for attosecond photoionization from an initial $p$ state can also be presented in terms of the Appell functions $F_{1}$.

\section{APPENDIX C: ROLE OF TERMS REPRESENTING PHOTON EMISSION}

We analyze here the contributions to the ionization probability of terms in the first- and second-order ionization amplitudes involving, respectively, one and two negative frequency components of the Fourier transform of the pulse, which are omitted in the approximate Eqs. (55) and (56). We rewrite the exact Eqs. (45) and (46) for the first- and secondorder transition amplitudes (which, we emphasize, were used to obtain all numerical results presented in this paper) as follows:

$$
\begin{aligned}
A_{1}=\widetilde{A}_{1}+\delta A_{1} & =e^{-i \phi}(\mathbf{e} \cdot \hat{\mathbf{p}}) \alpha_{+}+e^{i \phi}\left(\mathbf{e}^{*} \cdot \hat{\mathbf{p}}\right) \alpha_{-}, \\
A_{2}=\widetilde{A}_{2}+\delta A_{2}= & e^{-2 i \phi} \ell M_{0+2}^{++}+M_{0+2}^{+-}+e^{2 i \phi} \ell M_{0+2}^{--} \\
& -3\left[(\mathbf{e} \cdot \hat{\mathbf{p}})^{2} e^{-2 i \phi} M_{2}^{++}+|\mathbf{e} \cdot \hat{\mathbf{p}}|^{2} M_{2}^{+-}\right. \\
& \left.+\left(\mathbf{e}^{*} \cdot \hat{\mathbf{p}}\right)^{2} e^{2 i \phi} M_{2}^{--}\right]
\end{aligned}
$$

where $\widetilde{A}_{1}$ and $\tilde{A}_{2}$ are the approximate amplitudes defined in Eqs. (55) and (56), $\delta A_{1}=e^{i \phi}\left(\mathbf{e}^{*} \cdot \hat{\mathbf{p}}\right) \alpha_{-}$and $\delta A_{2}=e^{2 i \phi} \ell M_{0+2}^{--}$ $-3\left[\left(\mathbf{e}^{*} \cdot \hat{\mathbf{p}}\right)^{2} e^{2 i \phi} M_{2}^{--}\right]$. In terms of these contributions to the exact first- and second-order amplitudes, the ionization probability [cf. Eq. (38)] can be written as

$$
\begin{aligned}
\mathcal{W}= & p\left\{\left|A_{1}\right|^{2}+2 \operatorname{Re}\left[\left(A_{1}\right)^{*} A_{2}\right]+\left|A_{2}\right|^{2}\right\} \\
= & p\left\{\left|\widetilde{A}_{1}\right|^{2}+2 \operatorname{Re}\left\{\widetilde{A}_{1}^{*} \widetilde{A}_{2}\right\}+2 \operatorname{Re}\left\{\widetilde{A}_{1}^{*} \delta A_{1}\right\}+\left|\delta A_{1}\right|^{2}\right. \\
& +2 \operatorname{Re}\left\{\widetilde{A}_{1}^{*} \delta A_{2}\right\}+2 \operatorname{Re}\left[\left(\delta A_{1}\right)^{*} \widetilde{A}_{2}\right]+2 \operatorname{Re}\left[\left(\delta A_{1}\right)^{*} \delta A_{2}\right] \\
& \left.+\left|\widetilde{A}_{2}\right|^{2}+2 \operatorname{Re}\left[\left(\delta A_{2}\right)^{*} \widetilde{A}_{2}\right]+\left|\delta A_{2}\right|^{2}\right\} .
\end{aligned}
$$

The first two terms in Eq. $(\mathrm{C} 3),\left|\widetilde{A}_{1}\right|^{2}+2 \operatorname{Re}\left\{\tilde{A}_{1}^{*} \widetilde{A}_{2}\right\}$, are the largest ones and are the only ones taken into account in the theoretical part of this paper. One observes that there are many additional terms, and thus it is important to evaluate their relative importance. Clearly, the approximate first-order amplitude, $\widetilde{A}_{1}$, which describes absorption of one photon, has the largest magnitude, followed by the approximate secondorder amplitude, $\widetilde{A}_{2}$, which describes absorption of two pho-

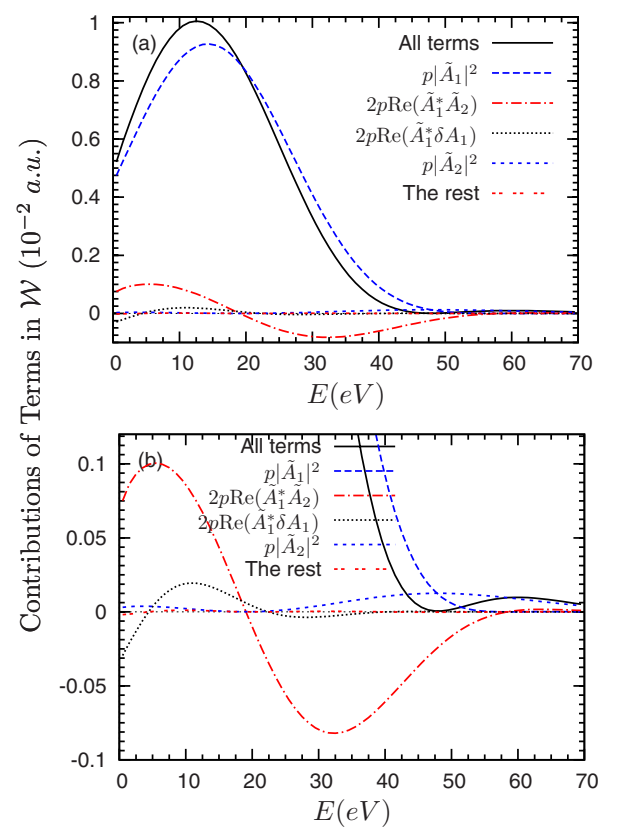

FIG. 11. (Color online) Contributions of different terms in Eq. (C3) to the probability $\mathcal{W}$ for ionization of the $\mathrm{H}$ atom with electrons ejected at an angle $\alpha=0$ by a linearly polarized, two-cycle, $\cos ^{2}$ pulse having $\omega=36 \mathrm{eV}, I_{0}=5 \times 10^{15} \mathrm{~W} / \mathrm{cm}^{2}$, and CEP $\phi=0$. Panel (b): same results as in panel (a), but on an enlarged scale.

tons plus absorption and emission of two photons. $\delta A_{1}$ describes emission of one photon; it is suppressed in comparison with the approximate amplitudes $\widetilde{A}_{1}$ and $\widetilde{A}_{2}$. The amplitude for emission of two photons, $\delta A_{2}$, has the smallest magnitude. It turns out, as we shall show, that of all the additional terms in Eq. (C3), only the terms $2 \operatorname{Re}\left\{\widetilde{A}_{1}^{*} \delta A_{1}\right\}$ and $\left|\widetilde{A}_{2}\right|^{2}$ are somewhat significant. To demonstrate this, we present in Fig. 11 the contributions of all terms in Eq. (C3). One sees that the contribution of the term $2 \operatorname{Re}\left\{\widetilde{A}_{1}^{*} \delta A_{1}\right\}$, which describes the interference of the one photon emission and one photon absorption amplitudes, gives a contribution of $\leq 5 \%$ at low final-state energies $\$ 20 \mathrm{eV}$. The two photon absorption term, $\left|\widetilde{A}_{2}\right|^{2} \sim I^{2}$, is somewhat significant in the region $40-70 \mathrm{eV}$, although, as mentioned in the theoretical section above, for high final-state energies, where the first-order transition amplitude is very small, one should take into account higher orders of PT, such as, e.g., the interference between the first- and third-order amplitudes, $\operatorname{Re}\left(A_{1}^{*} A_{3}\right)$, which also varies with intensity according to $\sim I^{2}$. However, the term $\operatorname{Re}\left(A_{1}^{*} A_{3}\right)$ can only be important in an energy region where both $A_{1}$ and $A_{3}$ are significant, which is the energy region of one photon absorption (for the first-order amplitude) and of absorption of two photons accompanied by emission of one photon (for the third-order amplitude). In the region of two photon absorption, it is expected that $\left|\widetilde{A}_{2}\right|^{2}$ $\approx\left|A_{2}\right|^{2}$ remains dominant. In addition $\left|A_{2}\right|^{2}$ ensures positive values for the ionization probability, which may become negative without it [see the region above $50 \mathrm{eV}$ in Fig. 11(b)]. All other additional terms in Eq. (C3) are insignificant and are not visually different from zero on the scale of Fig. 11 . 
These considerations allow us to present an improved theoretical expression [compared to Eqs. (16a) and (17)] for the ionization probability in which the CEP, pulse polarization, and angular dependence are explicit:

$$
\begin{aligned}
\mathcal{W}= & p\left(\left|\widetilde{A}_{1}\right|^{2}+2 \operatorname{Re}\left\{\tilde{A}_{1}^{*} \widetilde{A}_{2}\right\}+2 \operatorname{Re}\left\{\widetilde{A}_{1}^{*} \delta A_{1}\right\}+\left|\widetilde{A}_{2}\right|^{2}\right) \\
= & \mathcal{W}_{1}+\operatorname{Re}\left\{e^{-i \phi}\left[\left(\gamma_{1}+\gamma_{2}|\mathbf{e} \cdot \hat{\mathbf{p}}|^{2}\right)(\mathbf{e} \cdot \hat{\mathbf{p}})+\gamma_{3} \ell\left(\mathbf{e}^{*} \cdot \hat{\mathbf{p}}\right)\right]\right\} \\
& +p\left|\tilde{A}_{2}\right|^{2}
\end{aligned}
$$

Note that Eq. (C4) contains the exact $\mathcal{W}_{1}$ [cf. Eq. (13)] and the second-order term $p\left|\widetilde{A}_{2}\right|^{2}$. For an initial $s$ state

$$
\beta_{1}=0, \quad \beta_{2}=2 p \alpha_{+} \alpha_{-}^{*},
$$

while the other dynamical parameters are defined in Eqs. (57)-(60), respectively. The parametrization of the term $\left|\widetilde{A}_{2}\right|^{2}$ follows from Eq. (56); it involves the CEP dependence through the factors $\cos 2 \phi$ and $\sin 2 \phi$. For brevity, we present the results for only the two most important cases: for linear polarization,

$$
\left|\tilde{A}_{2}\right|_{l i n}^{2}=\left|l^{+}\right|^{2}+\left|l^{-}\right|^{2}+2 \operatorname{Re}\left\{e^{-2 i \phi} l^{+} l^{-*}\right\}
$$

where

$$
l^{+}=M_{0+2}^{++}-3 M_{2}^{++} \cos ^{2} \alpha, \quad l^{-}=M_{0+2}^{+-}-3 M_{2}^{+-} \cos ^{2} \alpha,
$$

and for circular polarization,

$$
\left|\widetilde{A}_{2}\right|_{c i r c}^{2}=\left|c^{+}\right|^{2}+\left|c^{-}\right|^{2}-2 \operatorname{Re}\left\{e^{-2 i(\xi \varphi-\phi)} c^{+} c^{-*}\right\},
$$

where

$$
c^{+}=(3 / 2) M_{2}^{++} \sin ^{2} \theta, \quad c^{-}=M_{0+2}^{+-}-(3 / 2) M_{2}^{+-} \sin ^{2} \theta .
$$

[1] T. Brabec and F. Krausz, Rev. Mod. Phys. 72, 545 (2000).

[2] G. Sansone et al., Science 314, 443 (2006).

[3] E. Goulielmakis et al., Science 320, 1614 (2008).

[4] F. Krausz and M. Ivanov, Rev. Mod. Phys. 81, 163 (2009).

[5] G. L. Yudin, S. Chelkowski, J. Itatani, A. D. Bandrauk, and P. B. Corkum, Phys. Rev. A 72, 051401(R) (2005).

[6] S. Chelkowski, G. L. Yudin, and A. D. Bandrauk, J. Phys. B 39, S409 (2006).

[7] T. Morishita, S. Watanabe, and C. D. Lin, Phys. Rev. Lett. 98, 083003 (2007).

[8] M. Uiberacker et al., Nature (London) 446, 627 (2007).

[9] J. Itatani, J. Levesque, D. Zeidler, H. P. H. Niikura, J. C. Kieffer, P. B. Corkum, and D. M. Villeneuve, Nature (London) 432, 867 (2004).

[10] A. D. Bandrauk, S. Chelkowski, and N. H. Shon, Phys. Rev. Lett. 89, 283903 (2002).

[11] E. Goulielmakis et al., Science 305, 1267 (2004).

[12] L. Y. Peng, E. A. Pronin, and A. F. Starace, New J. Phys. 10, 025030 (2008).

[13] L. A. A. Nikolopoulos and P. Lambropoulos, Phys. Rev. A 74, 063410 (2006).

[14] P. Lambropoulos, L. A. A. Nikolopoulos, M. G. Makris, and A. Mihelič, Phys. Rev. A 78, 055402 (2008).

[15] M. G. Makris, P. Lambropoulos, and A. Mihelič, Phys. Rev. Lett. 102, 033002 (2009).

[16] G. G. Paulus, F. Grasbon, H. Walther, P. Villoresi, M. Nisoli, S. Stagira, E. Priori, and S. De Silvestri, Nature (London) 414, 182 (2001).

[17] M. J. Abel, T. Pfeifer, A. Jullien, P. M. Nagel, M. J. Bell, D. M. Neumark, and S. R. Leone, J. Phys. B 42, 075601 (2009).

[18] T. Wittmann, B. Horvath, W. Helml, M. G. Schätzel, X. Gu, A. L. Cavalieri, G. G. Paulus, and R. Kienberger, Nat. Phys. 5, 357 (2009).

[19] E. Cormier and P. Lambropoulos, Eur. Phys. J. D 2, 15 (1998).

[20] D. B. Milošević, G. G. Paulus, D. Bauer, and W. Becker, J. Phys. B 39, R203 (2006).

[21] A. de Bohan, P. Antoine, D. B. Milošević, and B. Piraux, Phys. Rev. Lett. 81, 1837 (1998).
[22] T. Nakajima and S. Watanabe, Phys. Rev. Lett. 96, 213001 (2006).

[23] T. Nakajima and S. Watanabe, Opt. Lett. 31, 1920 (2006).

[24] V. Roudnev and B. D. Esry, Phys. Rev. Lett. 99, 220406 (2007)

[25] N. L. Manakov, S. I. Marmo, and A. V. Meremianin, J. Phys. B 29, 2711 (1996).

[26] N. L. Manakov, A. Maquet, S. I. Marmo, V. Veniard, and G. Ferrante, J. Phys. B 32, 3747 (1999).

[27] V. B. Berestetskii, E. M. Lifshitz, and L. P. Pitaevskii, Quantum Electrodynamics, 2nd ed. (Pegramon, Oxford, 1982), Sec. 8.

[28] J. Cooper and R. N. Zare, J. Chem. Phys. 48, 942 (1968).

[29] V. L. Jacobs, J. Phys. B 5, 2257 (1972).

[30] S. T. Manson and A. F. Starace, Rev. Mod. Phys. 54, 389 (1982).

[31] C. P. J. Martiny and L. B. Madsen, Phys. Rev. Lett. 97, 093001 (2006). Note that the terminology for left (right) circular polarization in this reference is different from the usual one, i.e., the electric fields for left (right) circular polarization in this reference correspond to ours for right (left) circular polarization.

[32] N. L. Manakov, M. V. Frolov, and A. F. Starace, J. Phys. B 36, R49 (2003), Sec. 1.1.

[33] N. L. Manakov and A. G. Fainshtein, Dokl. Akad. Nauk SSSR 244, 567 (1979) [Sov. Phys. Dokl. 44, 21 (1979)].

[34] N. L. Manakov and A. G. Fainshtein, Teor. Mat. Fiz. 48, 375 (1981) [Theor. Math. Phys. 48, 815 (1982)].

[35] R. M. Potvliege and R. Shakeshaft, Phys. Rev. A 41, 1609 (1990).

[36] C. P. J. Martiny, M. Abu-samha, and L. B. Madsen, J. Phys. B 42, 161001 (2009).

[37] H. Bateman and A. Erdélyi, Higher Transcendental Functions (McGraw-Hill, New York, 1953), Vol. 1.

[38] A. A. Krylovetsky, N. L. Manakov, and S. I. Marmo, Zh. Eksp. Teor. Fiz. 119, 45 (2001) [JETP 92, 37 (2001)].

[39] L. Y. Peng, F. Tan, Q. Gong, E. A. Pronin, and A. F. Starace, Phys. Rev. A 80, 013407 (2009). 